\title{
Subspace Correction Methods for a Class of Nonsmooth and Nonadditive Convex Variational Problems with Mixed $L^{1} / L^{2}$ Data-Fidelity in Image Processing*
}

\author{
Michael Hintermüller ${ }^{\dagger}$ and Andreas Langer ${ }^{\ddagger}$
}

\begin{abstract}
The minimization of a functional composed of a nonsmooth and nonadditive regularization term and a combined $L^{1}$ and $L^{2}$ data-fidelity term is proposed. It is shown analytically and numerically that the new model has noticeable advantages over popular models in image processing tasks. For the numerical minimization of the new objective, subspace correction methods are introduced which guarantee the convergence and monotone decay of the associated energy along the iterates. Moreover, an estimate of the distance between the outcome of the subspace correction method and the global minimizer of the nonsmooth objective is derived. This estimate and numerical experiments for image denoising, inpainting, and deblurring indicate that in practice the proposed subspace correction methods indeed approach the global solution of the underlying minimization problem.
\end{abstract}

Key words. subspace correction, domain decomposition, total variation minimization, convex optimization, image restoration, combined $L^{1} / L^{2}$ data-fidelity, convergence analysis, impulse noise, Gaussian noise, mixed noise

AMS subject classifications. 68U10, 94A08, 49M27, 65K10, 90C06

DOI. $10.1137 / 120894130$

1. Introduction. Subspace correction is a divide-and-conquer technique originally proposed for the numerical solution of partial differential equations. Algorithmically this is achieved by iteratively solving on each subspace an appropriately defined subproblem, which, in a variational setting, typically amounts to minimizing a smooth energy. For the overall algorithm, the convergence, rate of convergence, and independence of the rate of convergence from the mesh size of discretization are well established.

For nonsmooth problems, the resulting splitting algorithms still work fine as long as the energy splits additively with respect to the subspace decomposition. For such problems convergence and sometimes even rate of convergence are ensured; see, e.g., [27, 49]. Moreover, for image deblurring problems preconditioning effects of a specific subspace correction algorithm for minimizing a nonsmooth energy are shown in [51]. For nonsmooth and nonadditive energies, however, the research on subspace correction methods is far from being complete,

\footnotetext{
${ }^{*}$ Received by the editors October 8, 2012; accepted for publication (in revised form) June 26, 2013; published electronically October 30, 2013. This work was supported by the Austrian Science Fund FWF through the START Project Y 305-N18 "Interfaces and Free Boundaries" and the SFB Project F32 04-N18 "Mathematical Optimization and Its Applications in Biomedical Sciences," as well as by the German Research Fund (DFG) through the Research Center MATHEON Project C28 and the SPP 1253 "Optimization with Partial Differential Equations."

http://www.siam.org/journals/siims/6-4/89413.html

${ }^{\dagger}$ Department of Mathematics, Humboldt-University of Berlin, 10099 Berlin, Germany (hint@math.hu-berlin.de). This author's work was partly supported through a J. Tinsely Oden Fellowship at the Institute for Computational Engineering and Sciences (ICES) at UT Austin, TX

${ }^{\ddagger}$ Institute for Mathematics and Scientific Computing, University of Graz, A-8010 Graz, Austria (andreas.langer@ uni-graz.at).
} 
and for some problem classes counterexamples exist indicating failure of subspace correction; see, e.g., [28, 52].

From a computational point of view, one of the appeals of subspace correction methods is the fact that parallel algorithms can be devised which exploit the capabilities of multiprocessor or multicore computer architectures. Main advantages of associated iterative solvers include (i) dimension reduction; (ii) enhancement of parallelism; (iii) localized treatment of complex and irregular geometries, singularities, and anomalous regions; and sometimes (iv) reduction of the computational complexity of the underlying solution method. Among the important representatives of this algorithm class one finds the Jacobi method, the Gauss-Seidel method, point or block relaxation methods, multigrid methods, and domain decomposition methods. For further details on subspace correction and associated solvers, we refer the reader to [54].

In this paper we focus on subspace correction methods for a class of nonsmooth and nonadditive problems which arise in mathematical image processing. In this area the importance of devising such methods is clearly motivated by the continuous improvement of imaging hardware, which allows one to increase resolutions or to acquire vast amounts of data. In the context of variational methods in image processing, this may lead to extremely large-scale problems which need to be processed routinely.

In image restoration, the nonsmooth and nonadditive total variation (TV), proposed in [45] for image denoising, plays a fundamental role as a regularization technique, since it preserves edges and discontinuities in images. In this context, one typically minimizes an energy that consists of a data-fidelity term, which enforces the consistency between the recovered and the measured image, and the total variation as the regularization term. The choice of the data term usually depends on the type of noise contained in the measured image data. In this vein, for images corrupted by Gaussian noise a quadratic $L^{2}$ data-fidelity term has been successfully used in first order methods (see, e.g., [11, 12, 13, 17, 19, 20, 21, 22, 24, 32, 40, 43, 53, 57]) as well as in second order methods (see, e.g., [34]). In this approach, which we refer to as the $L^{2}$-TV model, the image $u$ is recovered from the observed data $g$ by solving

$$
\min _{u} \alpha\|T u-g\|_{L^{2}(\Omega)}^{2}+|D u|(\Omega),
$$

where $\Omega \subset \mathbb{R}^{2}$ is an open bounded set with Lipschitz boundary, $T$ is a bounded linear operator modeling the image-formation device (if the image is only corrupted by noise, one sets $T=I$ ), and $\alpha>0$ is a parameter. We recall that for $u \in L^{1}(\Omega)$

$$
V(u, \Omega):=\sup \left\{\int_{\Omega} u \operatorname{div} \phi d x: \phi \in\left[C_{c}^{1}(\Omega)\right]^{2},\|\phi\|_{\infty} \leq 1\right\}
$$

is the variation of $u$. In the event that $V(u, \Omega)<\infty$ we denote $|D u|(\Omega)=V(u, \Omega)$ and call it the total variation of $u$ in $\Omega$; see [2] for more details. If $u \in W^{1,1}(\Omega)$, then $|D u|(\Omega)=$ $\int_{\Omega}|\nabla u| d x$. The $L^{2}$-TV model usually does not yield a satisfactory restoration in the presence of salt-and-pepper noise, where the noisy image $g$, assumed throughout to have a dynamic range of $c_{\min } \leq g \leq c_{\max }$, is given by

$$
g(x)= \begin{cases}c_{\min } & \text { with probability } p_{1} \in[0,1), \\ c_{\max } & \text { with probability } p_{2} \in[0,1), \\ u(x) & \text { with probability } 1-p_{1}-p_{2}\end{cases}
$$


with $1-p_{1}-p_{2}>0$ [15]. Here, $p_{1}+p_{2}$ defines the noise level. Recently a nonsmooth $L^{1}$ data-fidelity term was suggested in [1], which treats impulse noise (e.g., salt-and-pepper noise) more successfully than a quadratic $L^{2}$ data term [41, 42, 25]; i.e., instead of (1.1), one considers

$$
\min _{u} \alpha\|T u-g\|_{L^{1}(\Omega)}+|D u|(\Omega),
$$

which we call the $L^{1}$-TV model.

In the case of simultaneous Gaussian and salt-and-pepper noise the choice of the datafidelity is unclear, and the literature on this subject appears rather scarce. In order to accommodate such situations, a two-phase reconstruction approach is suggested in [9]. In fact, in the first phase (most of) the outliers are detected, and in the second phase a variational functional consisting of a Mumford-Shah regularizer, which renders the problem nonconvex, is minimized. In contrast to this development we tackle the problem of removing simultaneous Gaussian and salt-and-pepper noise by optimizing a convex functional with a total variation regularizer and a combination of a quadratic $L^{2}$-term and a nonsmooth $L^{1}$-term. It turns out in our numerical experiments that such a combined data-fidelity term well suits the restoration task; see Figure 2 below. Analytically we show by means of an explicit example that the minimization of the newly proposed functional has noticeable advantages over the standard functionals, i.e., the $L^{2}$-TV and $L^{1}$-TV models. Algorithmically, we adapt the approach in [4], which was originally proposed for solving the $L^{1}-\mathrm{TV}$ model only, to our case of a combined data-fidelity term.

As all of the aforementioned solvers for TV-minimization are confined to small- and medium-scale problems only, we propose and analyze subspace correction, domain decomposition, and coordinate descent methods, as these are fundamental for reducing the overall problem to a finite number of subproblems, each of a size manageable for the above TV solvers.

Recently, in [28, 29, 30], nonoverlapping and overlapping domain decomposition strategies were introduced for solving the $L^{2}$-TV problem. In this context, the major difficulty lies in the correct treatment of the interfaces of the domain decomposition patches, i.e., the preservation of crossing discontinuities and the correct matching where the solution is continuous. We emphasize that well-known approaches such as those in $[10,18,46,47]$ are not directly applicable to the nonsmooth and nonadditive $L^{2}$-TV problem. In $[29,30]$ the convex objective under some linear constraint, ensuring the correct treatment of the internal interfaces, was iteratively minimized on each subdomain. While in these two papers an implementation guaranteeing convergence and monotonic decay of the objective energy is provided, convergence to the global minimizer of the $L^{2}-\mathrm{TV}$ problem cannot be ensured, in general. For one-dimensional problems, in [29] a proof is presented which establishes convergence of the overlapping domain decomposition algorithm to the global solution. We note that, although this proof is carried out for any finite-dimensional space, it is not yet clear how to prove convergence to the expected minimizer without further (and practically possibly critical) assumptions on the overlapping region for higher dimensions $(d>1)$.

In [28] a wavelet decomposition method is presented with properties similar to those of the aforementioned nonoverlapping domain decomposition methods. In that paper, an additional condition is imposed which allows one to establish global optimality of the limit point obtained by the domain decomposition method. Unfortunately, despite the good practical behavior of 
the method, this condition cannot be ensured to hold in general, as counterexamples have shown. Thus, with the aforementioned condition one can only check a posteriori whether the algorithm found the global minimizer or failed to do so. Moreover, no error estimates are available.

In the present paper we generalize the subspace correction strategy to more general functionals, which consist of a nonsmooth and nonadditive regularization term and a weighted combination of an $L^{1}$-term and a quadratic $L^{2}$-term; see (2.1) below. In this setting, the $L^{2}$-TV model considered in $[28,29,30]$ and the $L^{1}$-TV model are special instances. Note that the methods in $[28,29,30]$ differ from our approach. In fact, in $[28,29,30]$ each subspace minimization problem is approximated by a surrogate functional minimization, while we are minimizing on each subspace the exact subspace minimization problem. Thus, a different convergence analysis is required. Similarly to the domain decomposition methods in [29, 30], we are able to show that our subspace correction methods for the newly introduced functional are guaranteed to converge and to monotonically decrease the energy. In addition, we are able to establish an estimate of the distance of the limit point obtained from the subspace correction method to the true global minimizer. With the help of this estimate, we demonstrate in our numerical experiments that the sequence generated by our proposed algorithm indeed approaches the global minimizer of the objective functional.

The rest of the paper is organized as follows: In section 2 we state the problem of interest, and we propose a solution method for the global minimization problem. Moreover, we motivate the choice of the objective functional by analyzing theoretically an illustrative example as well by numerical experiments. Our alternating and parallel subspace correction methods are introduced in section 3 in a Banach space setting where we also state some convergence properties. In section 4 we describe the problem in a discrete setting and show optimality properties of our subspace correction methods which allow us to estimate the distance of a limit point obtained by subspace correction to the minimizer of the total energy. In section 5 we present our subspace correction methods for the special cases of overlapping and nonoverlapping domain decomposition. Details on the implementation of the solvers for the domain decomposition methods are provided. Finally we show sequential and parallel numerical experiments for TV minimization.

2. Image restoration with mixed $L^{1} / L^{2}$ data-fidelity. We are interested in solving the following minimization problem:

$$
\min _{u \in L^{2}(\Omega)} J_{\alpha_{1}, \alpha_{2}}(u):=\alpha_{1}\left\|T_{1} u-g_{1}\right\|_{L^{1}(\Omega)}+\alpha_{2}\left\|T_{2} u-g_{2}\right\|_{L^{2}(\Omega)}^{2}+\varphi(|D u|)(\Omega),
$$

where $T_{i}: L^{2}(\Omega) \rightarrow L^{2}(\Omega)$ is a bounded linear operator, $g_{i} \in L^{2}(\Omega)$ is a given datum, $\alpha_{i} \geq 0$ for $i=1,2$, with $\alpha_{1}+\alpha_{2}>0, \Omega \subset \mathbb{R}^{d}, d \in \mathbb{N}$, and $\varphi(|\cdot|)$ is a convex function of measures representing regularization.

We assume that $\left\|T_{i}\right\|<1$ for $i=1,2$, which is not at all a restriction, as a proper rescaling of the problem reestablishes the desired setting whenever a norm exceeds 1 . In what follows we make the assumption that $J_{\alpha_{1}, \alpha_{2}}$ is bounded from below and coercive, i.e., $\left\{J_{\alpha_{1}, \alpha_{2}} \leq C\right\}=\left\{u \in L^{2}(\Omega): J_{\alpha_{1}, \alpha_{2}}(u) \leq C\right\}$ is bounded in $L^{2}(\Omega)$ for all constants $C>0$, in order to guarantee that problem (2.1) has solutions. Moreover we assume that

$\left(A_{\varphi}\right) \varphi: \mathbb{R} \rightarrow \mathbb{R}$ is a convex function, nondecreasing in $\mathbb{R}^{+}$with 
(i) $\varphi(0)=0$;

(ii) $c z-b \leq \varphi(z) \leq c z+b$ for all $z \in \mathbb{R}^{+}$for some constant $c>0$ and $b \geq 0$.

Note that for the particular example $\varphi(t)=t$, the third term in (2.1) becomes the wellknown total variation of $u$ in $\Omega$, and we then call (2.1) the $L^{1}-L^{2}$-TV model. Other functions which fulfill assumption $\left(A_{\varphi}\right)$ are $\varphi(t)=\sqrt{1+t^{2}}-1$ (the function of minimal surfaces) and $\varphi(t)=\log \cosh t[50]$.

2.1. Qualitative behavior of the $L^{1}-L^{2}-T V$ model. In order to motivate our proposed model (2.1), we use a simple and illustrative example in two dimensions, where $\varphi(t)=t$, which we compare with the $L^{1}$-TV model (i.e., when $\alpha_{2}=0$ in (2.1)) and with the $L^{2}$-TV model (i.e., when $\alpha_{1}=0$ in (2.1)).

Example 2.1. Let the observed image $g_{1}=g_{2}$ be the characteristic function $1_{B_{r}(0)}$ of a disk $B_{r}(0)$ centered at the origin with radius $r>0$. We are interested in the explicit solution of the problem in (2.1) when $\Omega=\mathbb{R}^{2}, T_{1}=T_{2}=I$ is the identity operator, and $\varphi(t)=t$ for three different cases, (i) $\alpha_{1}=0, \alpha_{2}>0\left(L^{2}-T V\right)$, (ii) $\alpha_{1}>0, \alpha_{2}=0\left(L^{1}-T V\right)$, (iii) $\alpha_{1}>0$, $\alpha_{2}>0\left(L^{1}-L^{2}-T V\right)$.

For the first two cases we recall the solutions found in [16, 39]:

(i) For $\alpha_{1}=0, \alpha_{2}>0$ the unique minimizer $u_{0, \alpha_{2}}$ is given by

$$
u_{0, \alpha_{2}}= \begin{cases}0 & \text { if } 0 \leq r<\frac{1}{\alpha_{2}}, \\ \left(1-\frac{1}{\alpha_{2} r}\right) 1_{B_{r}(0)} & \text { if } r \geq \frac{1}{\alpha_{2}} .\end{cases}
$$

(ii) For $\alpha_{1}>0, \alpha_{2}=0$ a minimizer $u_{\alpha_{1}, 0}$ is given by

$$
u_{\alpha_{1}, 0} \in \begin{cases}\{0\} & \text { if } 0 \leq r<\frac{2}{\alpha_{1}}, \\ \left\{c 1_{B_{r}(0)}: c \in[0,1]\right\} & \text { if } r=\frac{2}{\alpha_{1}}, \\ \left\{1_{B_{r}(0)}\right\} & \text { if } r>\frac{2}{\alpha_{1}} .\end{cases}
$$

(iii) For $\alpha_{1}, \alpha_{2}>0$ one can reason that every minimizer has to be of the form $c 1_{B_{r}(0)}$ for $c \in[0,1]$. Therefore we just need to minimize the function

$$
J_{\alpha_{1}, \alpha_{2}}\left(c 1_{B_{r}(0)}\right)=\alpha_{1} \pi r^{2}|1-c|+\alpha_{2} \pi r^{2}(1-c)^{2}+2 \pi r c
$$

over $c \in[0,1]$. Then the optimality condition for $c$ is given by

$$
-\alpha_{1} \pi r^{2}-2 \alpha_{2} \pi r^{2}(1-c)+2 \pi r=0,
$$

which is equivalent to

$$
c=\frac{2 \alpha_{2}+\alpha_{1}}{2 \alpha_{2}}-\frac{1}{\alpha_{2} r} .
$$

Hence, the unique minimizer is given by

$$
u_{\alpha_{1}, \alpha_{2}}= \begin{cases}0 & \text { if } 0 \leq r<\frac{2}{2 \alpha_{2}+\alpha_{1}}, \\ \left(\frac{2 \alpha_{2}+\alpha_{1}}{2 \alpha_{2}}-\frac{1}{\alpha_{2} r}\right) 1_{B_{r}(0)} & \text { if } \frac{2}{2 \alpha_{2}+\alpha_{1}} \leq r \leq \frac{2}{\alpha_{1}}, \\ 1_{B_{r}(0)} & \text { if } r>\frac{2}{\alpha_{1}} .\end{cases}
$$


From this example we clearly see the difference between the $L^{2}$-TV model and the $L^{1}$-TV model. When the $L^{1}$-fidelity is used, then the solution is constant except at a special value $\left(r=\frac{2}{\alpha_{1}}\right)$, where it undergoes a sudden transition. When in addition to the $L^{1}$-fidelity the $L^{2}$-term is also present, then the solution is constant except in an interval $\left(\frac{2}{2 \alpha_{2}+\alpha_{1}} \leq r \leq \frac{2}{\alpha_{1}}\right)$, where it experiences a smooth transition. In contrast, when only the $L^{2}$-fidelity plus a TVterm is used, then the solution is only constant for $0 \leq r<\frac{2}{\alpha_{2}}$ and hyperbolically increasing otherwise.

The differences between the $L^{2}-\mathrm{TV}$ model and the $L^{1}$-TV model result in the following observation: Fix $\alpha_{1}=\alpha_{2}=\alpha>0$ and set, as in Example 2.1, $g_{1}=g_{2}=1_{B_{r}(0)}$ and $T_{1}=T_{2}=I$. Then the solution $u_{0, \alpha}$ of the $L^{2}$-TV model is identically 0 if $r<\frac{1}{\alpha}$. This is clearly an advantage over the $L^{1}$-TV model, where $u_{\alpha, 0}=0$ if $r<\frac{2}{\alpha}$, since smaller features can be maintained with the $L^{2}$-TV model. In contrast, the $L^{2}$-TV model is not able to preserve the original features perfectly (except if $\alpha=\infty$ ) but obtains them only with a loss of energy; i.e., $u_{0, \alpha}=\left(1-\frac{1}{\alpha r}\right) 1_{B_{r}(0)}$ if $r \geq \frac{1}{\alpha}$. This is different for the $L^{1}$-TV model, where we have that $u_{\alpha, 0}=1_{B_{r}(0)}$ if $r>\frac{2}{\alpha}$, and hence features can be perfectly preserved. This is naturally a clear advantage of the latter model.

For the combined $L^{1}-L^{2}$-TV model, we observe that $u_{\alpha, \alpha}=0$ if $0 \leq r<\frac{2}{3 \alpha}$, and hence even smaller features as with the $L^{2}$-TV model can be maintained. But still we are able to preserve original features perfectly as in the $L^{1}$-TV model. Moreover, the transition between the just mentioned constant states is smooth, which is clearly a property coming from the $L^{2}$-term, which renders the solution unique.

2.2. Practical behavior. We first specify a solution algorithm for the model in (2.1) and then study the quantitative behavior of this model and the proposed method by means of a benchmark example.

A solution algorithm. For computing a minimizer of the global problem in (2.1) we suggest an algorithm which is an adaptation of a method that was originally proposed for $L^{1}$-TV minimization problems in [4]. For this purpose we replace $J_{\alpha_{1}, \alpha_{2}}$ by the functional

$$
\alpha_{1}\|v\|_{L^{1}(\Omega)}+\frac{1}{2 \gamma}\left\|T_{1} u-g_{1}-v\right\|_{L^{2}(\Omega)}^{2}+\alpha_{2}\left\|T_{2} u-g_{2}\right\|_{L^{2}(\Omega)}^{2}+\varphi(|D u|)(\Omega),
$$

where $\gamma>0$ is small, so that we have $g_{1} \approx T_{1} u-v$. Actually, for $\gamma \rightarrow 0,(2.2)$ approaches the objective functional in (2.1). Now we minimize (2.2) with respect to $u$ and $v$, which we perform in the following alternating way:

(1) For fixed $u$ solve

$$
\min _{v \in L^{2}(\Omega)} \alpha_{1}\|v\|_{L^{1}(\Omega)}+\frac{1}{2 \gamma}\left\|T_{1} u-g_{1}-v\right\|_{L^{2}(\Omega)}^{2} .
$$

The minimizer $v^{*}$ of $(2.3)$ can be easily computed via a soft-thresholding, i.e., $v^{*}=$ $\mathrm{ST}\left(T_{1} u-g_{1}, \gamma \alpha_{1}\right)$, where

$$
\operatorname{ST}(g, \beta)(x)= \begin{cases}g(x)-\beta & \text { if } g(x)>\beta \\ 0 & \text { if }|g(x)| \leq \beta \\ g(x)+\beta & \text { if } g(x)<-\beta\end{cases}
$$


for all $x \in \Omega$.

(2) For fixed $v$ solve

$$
\min _{u \in L^{2}(\Omega)} \frac{1}{2 \gamma}\left\|T_{1} u-g_{1}-v\right\|_{L^{2}(\Omega)}^{2}+\alpha_{2}\left\|T_{2} u-g_{2}\right\|_{L^{2}(\Omega)}^{2}+\varphi(|D u|)(\Omega) .
$$

This step is realized on the surrogate functional

$$
\begin{aligned}
S(u, a):= & \frac{1}{2 \gamma}\left\|T_{1} u-g_{1}-v\right\|_{L^{2}(\Omega)}^{2}+\alpha_{2}\left\|T_{2} u-g_{2}\right\|_{L^{2}(\Omega)}^{2}+\varphi(|D u|)(\Omega) \\
& +\frac{1}{2 \gamma}\left(\|u-a\|_{L^{2}(\Omega)}^{2}-\left\|T_{1}(u-a)\right\|_{L^{2}(\Omega)}^{2}\right) \\
& +\alpha_{2}\left(\|u-a\|_{L^{2}(\Omega)}^{2}-\left\|T_{2}(u-a)\right\|_{L^{2}(\Omega)}^{2}\right) \\
= & \frac{1}{2 \gamma}\left\|u-z_{1}\right\|_{L^{2}(\Omega)}^{2}+\alpha_{2}\left\|u-z_{2}\right\|_{L^{2}(\Omega)}^{2}+\varphi(|D u|)(\Omega)+\psi,
\end{aligned}
$$

with $a, u \in L^{2}(\Omega)$ and where $z_{1}=z_{1}(a)=a+T_{1}^{*}\left(g_{1}+v-T_{1} a\right), z_{2}=z_{2}(a)=$ $a+T_{2}^{*}\left(g_{2}-T_{2} a\right)$, and $\psi$ is a function independent of $u$. Note that

$$
\min _{u \in L^{2}(\Omega)} S(u, a) \Leftrightarrow \min _{u \in L^{2}(\Omega)}\left\|u-\frac{\gamma}{1+2 \alpha_{2} \gamma}\left(\frac{1}{\gamma} z_{1}+2 \alpha_{2} z_{2}\right)\right\|_{L^{2}(\Omega)}^{2}+\frac{2 \gamma}{1+2 \alpha_{2} \gamma} \varphi(|D u|)(\Omega) .
$$

For $\varphi(t)=t,(2.6)$ is a variant of the Rudin-Osher-Fatemi (ROF)-problem [45]. There exist several numerical methods for solving the ROF-problem efficiently; see, for example, $[11,17,19,22,24,32,33,34,40,43]$. Hence an approximate solution of (2.5) can be computed by the following iterative algorithm: Initialize $u^{(0)} \in L^{2}(\Omega)$ and iterate

$$
u^{(\ell+1)}=\arg \min _{u \in L^{2}(\Omega)} S\left(u, u^{(\ell)}\right), \quad \ell \geq 0 .
$$

If $T_{1}=T_{2}=I$ and $\varphi(t)=t$, then (2.5) becomes the ROF-problem, i.e.,

$$
\min _{u \in L^{2}(\Omega)}\left\|u-\frac{\gamma}{1+2 \alpha_{2} \gamma}\left(\frac{1}{\gamma}\left(g_{1}+v\right)+2 \alpha_{2} g_{2}\right)\right\|_{L^{2}(\Omega)}^{2}+\frac{2 \gamma}{1+2 \alpha_{2} \gamma}|D u|(\Omega),
$$

which can be solved directly by means of the aforementioned methods.

Numerical examples. In Example 2.1 above we compute the exact solution of the minimization problem $(2.1)$ with $\varphi(t)=t$. There we show that the newly proposed $L^{1}-L^{2}-\mathrm{TV}$ model preserves the original signal better than do either the $L^{1}$-TV or $L^{2}$-TV models. In this section we support this result by numerical computations for different choices of $\alpha_{1}$ and $\alpha_{2}$ in (2.1) for $\varphi(t)=t$ and for a given noisy image $g\left(=g_{1}=g_{2}\right)$, which is specified below. Note that the dynamic range of all image data considered in this paper is $\left[c_{\min }, c_{\max }\right]:=[0,1]$. As a comparison for the different restoration qualities of the image we use the PSNR (peak signal-to-noise ratio) given by

$$
\operatorname{PSNR}=20 \log \frac{1}{\left\|u_{\text {org }}-u^{*}\right\|},
$$


where $u_{\text {org }}$ denotes the original image before any corruption and $u^{*}$ the restored image. In general, when comparing PSNR-values, large values indicate a better reconstruction than smaller values.

The chosen test image $u_{\text {org }}$, shown in Figure 1(a), consists of squares of various sizes. We are interested in selecting $\alpha_{i}, i=1,2$, such that the original image $u_{\text {org }}$ is preserved best. Therefore we compute the minimizer of the $L^{1}-L^{2}$-TV model with $g=u_{\text {org }}$ and $T_{i}=I$, $i=1,2$, for $\alpha_{1}, \alpha_{2} \in\{0,0.1,0.2, \ldots, 0.9,1,1.5\}$ and depict the obtained PSNR values in Figure 1(b). Note that for $\alpha_{1}=\alpha_{2}=0$ we set the PSNR value to the default value of 0 . For $\alpha_{2}=0$ and $\alpha_{1}>0$ we see the typical behavior of the $L^{1}$-TV model. In fact, depending on the size of $\alpha_{1}$, different scales of the image features are preserved exactly. Typically, for decreasing $\alpha_{1}$, features at smaller scales are suddenly "lost" in the reconstruction, whereas other features are still recovered perfectly. Also the fading-away effect of image features at various scales depending on the decreasing choice of $\alpha_{2}$ of the $L^{2}$-TV model can be seen clearly. However, Figure 1(b) shows that a combination of $\alpha_{1}>0, \alpha_{2}>0$ always gives a better restoration than setting one of the parameters to 0 .

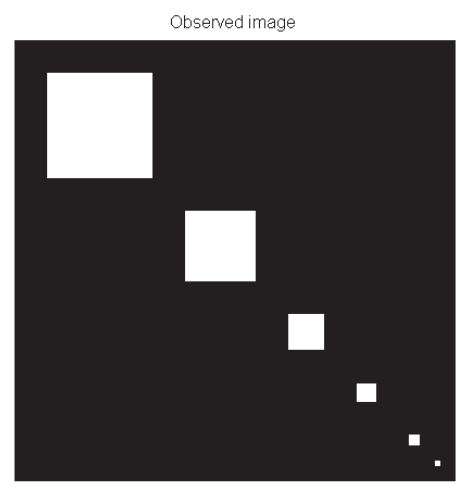

(a)

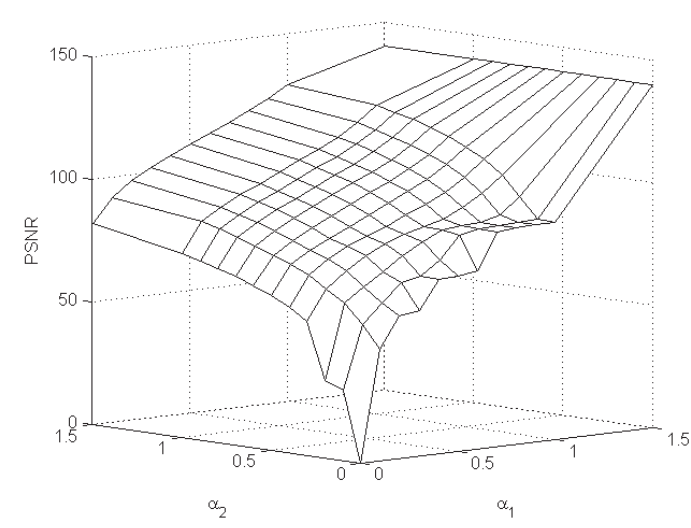

(b)

Figure 1. (a) Phantom image. (b) PSNR-values of the scale space generated by minimizing the $L^{1}-L^{2}-T V$ model for different choices of the parameters $\alpha_{1}$ and $\alpha_{2}$.

In the second experiment we corrupt the original image of Figure 1(a) by Gaussian noise and salt-and-pepper noise; i.e., $g$ is now the image in Figure 2(a). Then we again compute the minimizer of the $L^{1}-L^{2}$-TV model for $\alpha_{1}, \alpha_{2} \in\{0,0.1,0.2, \ldots, 1\}$ and depict the obtained PSNR values in Figure 2(b). The maximal PSNR value is reached for $\alpha_{1}=0.7$ and $\alpha_{2}=0.4$, which shows that for a combination of these two noise types the $L^{1}-L^{2}$-TV model outperforms the $L^{1}$-TV model as well as the $L^{2}$-TV model.

For the sake of a performance reference we also compare our $L^{1}-L^{2}-\mathrm{TV}$ minimization algorithm with the frequently used ROAD-trilateral filter [31], which is designed to remove a mixture of Gaussian noise (with zero mean and variance $\sigma$ ) and impulse noise. This filter is based on a simple statistic to detect outliers in an image. For our comparison we restore the Barbara image (see Figure 3(a)) and the Cameraman image (see Figure 3(b)) for saltand-pepper noise with $p_{1}=p_{2} \in\{0.05,0.15\}$ and low levels of Gaussian noise, i.e., $\sigma \in$ 


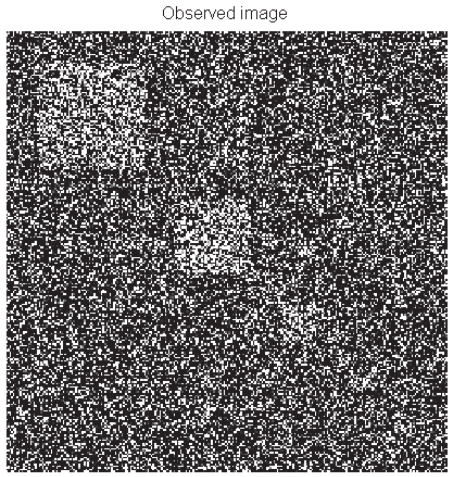

(a)

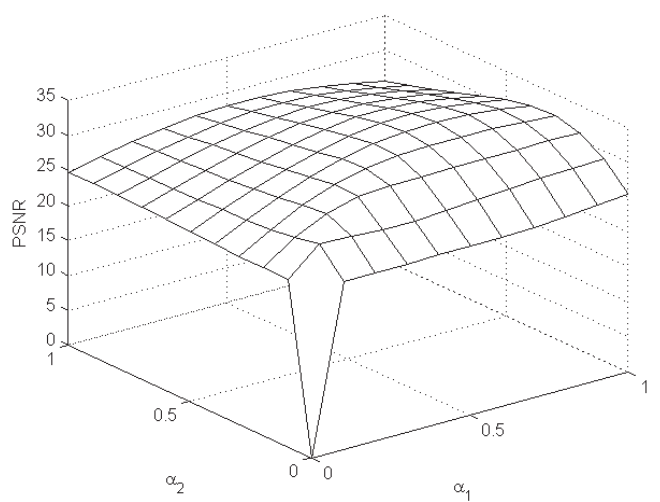

(b)

Figure 2. (a) Image of Figure 1 corrupted by Gaussian noise (with zero mean and variance $\sigma=0.1$ ) and $75 \%$ salt-and-pepper noise (more precisely, $p_{1}=0.5$ and $p_{2}=0.25$ ). (b) PSNR-values of the minimizer of the $L^{1}-L^{2}-T V$ model for different choices of the parameters $\alpha_{1}$ and $\alpha_{2}$.

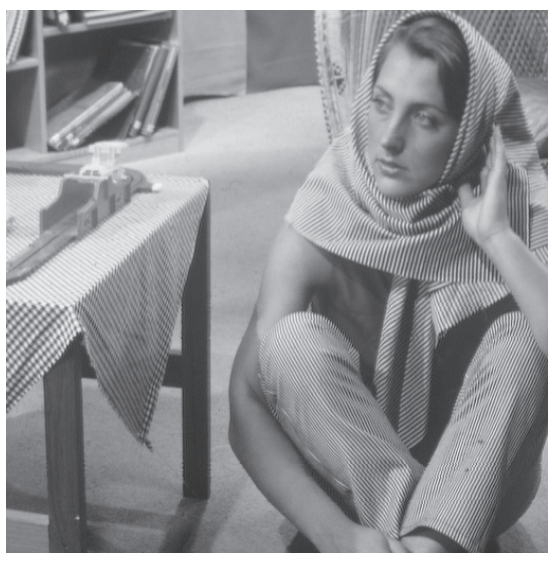

(a)

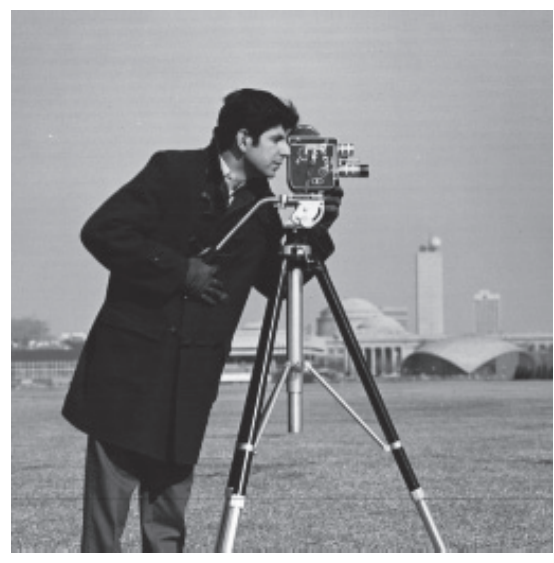

(b)

Figure 3. (a) The original Barbara image of size $512 \times 512$ pixels. (b) The original Cameraman image of size $256 \times 256$ pixels.

$\{5 / 255,15 / 255\}$, as available in the literature. Here we further test the algorithms for higher levels of Gaussian noise, i.e., $\sigma \in\{\sqrt{0.02}, 0.2\}$, plus salt-and-pepper noise; see Table 1 . We also note that for impulse noise-dominated contamination of image data the implementation of strategies, such as the one in [14] and the references therein, enhances the performance of the algorithm. In the $L^{1}-L^{2}$-TV minimization algorithm we set $T_{i}=I, i=1,2, \alpha_{1} \in$ $\{0.1,0.4,0.7,1,1.3,1.6,2\}$, and $\alpha_{2} \in\{0,0.1,0.4,0.7,1\}$. For the ROAD-trilateral filter we choose $\sigma_{S}=1, \sigma_{I}=40 / 255, \sigma_{J}=30 / 255$, and $\sigma_{R}$ is optimized between $10 / 255$ and $50 / 255$ as suggested in [23] with window-size $3 \times 3$. In Table 1 we show the highest PSNR-values achieved in our experiments for both methods. We observe that the $L^{1}-L^{2}-\mathrm{TV}$ minimization 
Table 1

PSNR results for the $512 \times 512$ pixel image "Barbara" and the $256 \times 256$ pixel image "Cameraman." The parameters of the ROAD-trilateral filter are $\sigma_{S}=1, \sigma_{I}=40 / 255, \sigma_{J}=30 / 255$, and $\sigma_{R}$ is optimized between $10 / 255$ and 50/255, as suggested in [23]. For the $L^{1}-L^{2}-T V$ minimization algorithm we show in parentheses the parameters for which the best PSNR is obtained.

\begin{tabular}{|c|c|c|c|c|}
\hline & $\sigma$ & $p_{1}=p_{2}$ & ROAD-trilateral & $L^{1}-L^{2}-\mathrm{TV}$ \\
\hline \multirow{8}{*}{ Barbara } & $5 / 255$ & $\begin{array}{l}0.05 \\
0.15\end{array}$ & $\begin{array}{l}24.23 \\
22.03\end{array}$ & $\begin{array}{l}26.01\left(\alpha_{1}=1.3, \alpha_{2}=1\right) \\
24.56\left(\alpha_{1}=1.3, \alpha_{2}=0\right)\end{array}$ \\
\hline & $15 / 255$ & 0.05 & 23.61 & $25.06\left(\alpha_{1}=1.6, \alpha_{2}=0\right)$ \\
\hline & $\sqrt{0.02}$ & 0.10 & $\frac{21.02}{22.19}$ & $\frac{23,78\left(\alpha_{1}=1.3, \alpha_{2}=0\right)}{23.18\left(\alpha_{1}=0.7, \alpha_{2}=0.4\right)}$ \\
\hline & & 0.15 & 18.50 & $22.59\left(\alpha_{1}=0.7, \alpha_{2}=0\right)$ \\
\hline & $\sqrt{0.02}$ & 0.005 & 22.51 & $23.66\left(\alpha_{1}=0.7, \alpha_{2}=1\right)$ \\
\hline & & 0.01 & 22.49 & $23.60\left(\alpha_{1}=0.7, \alpha_{2}=1\right)$ \\
\hline & 0.2 & 0.005 & 21.30 & $23.05\left(\alpha_{1}=0.4, \alpha_{2}=1\right)$ \\
\hline & & 0.01 & 21.26 & $23.05\left(\alpha_{1}=0.4, \alpha_{2}=1\right)$ \\
\hline \multirow{10}{*}{ Cameraman } & $5 / 255$ & 0.05 & 23.96 & $27.12\left(\alpha_{1}=1.3, \alpha_{2}=1\right)$ \\
\hline & & 0.15 & 21.83 & $25.01\left(\alpha_{1}=1.3, \alpha_{2}=0\right)$ \\
\hline & $15 / 255$ & 0.05 & 23.72 & $25.92\left(\alpha_{1}=1.3, \alpha_{2}=0.4\right)$ \\
\hline & & 0.15 & 21.31 & $23.98\left(\alpha_{1}=1.3, \alpha_{2}=0\right)$ \\
\hline & $\sqrt{0.02}$ & 0.05 & 22.02 & $23.49\left(\alpha_{1}=1, \alpha_{2}=0.1\right)$ \\
\hline & & 0.15 & 18.45 & $22.33\left(\alpha_{1}=1, \alpha_{2}=0\right)$ \\
\hline & $\sqrt{0.02}$ & 0.005 & 22.48 & $24.23\left(\alpha_{1}=0.7, \alpha_{2}=1\right)$ \\
\hline & & 0.01 & 22.44 & $24.25\left(\alpha_{1}=0.7, \alpha_{2}=1\right)$ \\
\hline & 0.2 & 0.005 & 22.13 & $23.29\left(\alpha_{1}=0.7, \alpha_{2}=0.7\right)$ \\
\hline & & 0.01 & 22.07 & $23.22\left(\alpha_{1}=0.7, \alpha_{2}=0.7\right)$ \\
\hline
\end{tabular}

algorithm outperforms the ROAD-trilateral filter with respect to PSNR and that $\alpha_{2}=0$ yields the best results in the case of relatively high impulse noise.

3. Subspace correction approach in $L^{2}(\Omega)$. In order to enable or to accelerate the solution process, subspace correction and domain decomposition methods offer the potential to split the computational workload and to solve (in parallel) a sequence of more tractable problems. In this sense we now follow the general philosophy of subspace correction and seek to minimize $J_{\alpha_{1}, \alpha_{2}}$ by decomposing $L^{2}(\Omega)$ into two appropriate subspaces $V_{1}$ and $V_{2}$ such that $L^{2}(\Omega)=V_{1}+V_{2}$. With this splitting we aim to solve (2.1) by the following alternating algorithm.

Choose an initial $u^{(0)}=: \tilde{u}_{1}^{(0)}+\tilde{u}_{2}^{(0)} \in V_{1}+V_{2}$, for example $u^{(0)}=0$, and iterate

$$
\left\{\begin{array}{l}
u_{1}^{(n+1)} \leftarrow \arg \min _{u_{1} \in V_{1}} J_{\alpha_{1}, \alpha_{2}}\left(u_{1}+\tilde{u}_{2}^{(n)}\right), \\
u_{2}^{(n+1)} \leftarrow \arg \min _{u_{2} \in V_{2}} J_{\alpha_{1}, \alpha_{2}}\left(u_{1}^{(n+1)}+u_{2}\right), \\
u^{(n+1)} \leftarrow u_{1}^{(n+1)}+u_{2}^{(n+1)}, \\
\tilde{u}_{1}^{(n+1)} \leftarrow \chi_{1} \cdot u^{(n+1)}, \\
\tilde{u}_{2}^{(n+1)} \leftarrow \chi_{2} \cdot u^{(n+1)},
\end{array}\right.
$$

where $\chi_{1}, \chi_{2} \in L^{\infty}(\Omega)$ have the properties (a) $\chi_{1}+\chi_{2}=1$ and (b) $\chi_{i} \in V_{i}$ for $i=1,2$. Let $\kappa:=\max \left\{\left\|\chi_{1}\right\|_{\infty},\left\|\chi_{2}\right\|_{\infty}\right\}<\infty$. Although $\tilde{u}_{1}^{(n+1)}$ is essentially not used in the above 
algorithm, it is present for theoretical reasons. From the assumptions on $\chi_{i}$ we obtain that $u^{(n)}=\left(\chi_{1}+\chi_{2}\right) u^{(n)}=\tilde{u}_{1}^{(n)}+\tilde{u}_{2}^{(n)}$. Further, if the $V_{i}$ 's are orthogonal, i.e., $L^{2}(\Omega)=V_{1} \oplus V_{2}$, then $\tilde{u}_{i}^{(n)}=u_{i}^{(n)}$ for all $n \in \mathbb{N}$, and hence in this case there is no need to introduce the variables $\tilde{u}_{i}^{(n)}$; cf. (5.2) below. The parallel version of the algorithm in (3.1) reads as follows.

Choose an initial $u^{(0)}=: \tilde{u}_{1}^{(0)}+\tilde{u}_{2}^{(0)} \in V_{1}+V_{2}$, for example $u^{(0)}=0$, and iterate

$$
\left\{\begin{aligned}
& u_{1}^{(n+1)} \leftarrow \arg \min _{u_{1} \in V_{1}} J_{\alpha_{1}, \alpha_{2}}\left(u_{1}+\tilde{u}_{2}^{(n)}\right), \\
& u_{2}^{(n+1)} \leftarrow \arg \min _{u_{2} \in V_{2}} J_{\alpha_{1}, \alpha_{2}}\left(\tilde{u}_{1}^{(n)}+u_{2}\right), \\
& u^{(n+1)} \leftarrow \frac{u_{1}^{(n+1)}+u_{2}^{(n+1)}+u^{(n)}}{2}, \\
& \tilde{u}_{1}^{(n+1)} \leftarrow \chi_{1} \cdot u^{(n+1)}, \\
& \tilde{u}_{2}^{(n+1)} \leftarrow \chi_{2} \cdot u^{(n+1)} .
\end{aligned}\right.
$$

We define the orthogonal complement of $V_{i}$ in $L^{2}(\Omega)$ by $V_{i}^{c}$, i.e., $L^{2}(\Omega)=V_{i} \oplus V_{i}^{c}$, and we define by $\pi_{V_{i}}$ the corresponding orthogonal projection onto $V_{i}$. Moreover, we define the domain of a functional $\mathcal{J}: L^{2}(\Omega) \rightarrow \overline{\mathbb{R}}$ as the set $\operatorname{Dom}(\mathcal{J})=\left\{v \in L^{2}(\Omega): \mathcal{J}(v) \neq \infty\right\}$.

Note that the subspace minimization problems in (3.1) and (3.2) can be written as constrained optimization problems of the form

$$
\min _{v \in L^{2}(\Omega)} J_{\alpha_{1}, \alpha_{2}}(v) \quad \text { subject to (s.t.) } A v=b,
$$

where $A: L^{2}(\Omega) \rightarrow L^{2}(\Omega)$ is a linear and continuous operator on $L^{2}(\Omega)$ and $b \in L^{2}(\Omega)$. In particular, we have

$$
\min _{v \in L^{2}(\Omega)} J_{\alpha_{1}, \alpha_{2}}(v+b) \quad \text { s.t. } \pi_{V_{i}^{c}} v=0
$$

or equivalently

$$
\min _{v \in L^{2}(\Omega)} J_{\alpha_{1}, \alpha_{2}}(v) \quad \text { s.t. } \pi_{V_{i}^{c}}(v)=\pi_{V_{i}^{c}}(b)
$$

where $b=u_{1}^{(n+1)}$ for the second minimization problem in (3.1) and $b=\tilde{u}_{j}^{(n)}$ for the first minimization problem in (3.1) and the minimization problems in (3.2) for $i=1,2$ and $j \in$ $\{1,2\} \backslash\{i\}$.

For any attainable $b \in V_{j}$, i.e., when there exists an $u \in \operatorname{Dom}\left(J_{\alpha_{1}, \alpha_{2}}\right)$ such that $\pi_{V_{i}^{c}}(u)=$ $\pi_{V_{i}^{c}}(b)$, we observe that $\left\{u \in L^{2}(\Omega): \pi_{V_{i}^{c}}(u)=\pi_{V_{i}^{c}}(b), J_{\alpha_{1}, \alpha_{2}}(u) \leq C\right\} \subset\left\{J_{\alpha_{1}, \alpha_{2}} \leq C\right\}$ for all $C>0, i=1,2$, and $j \in\{1,2\} \backslash\{i\}$. Hence the former set is bounded by the coercivity assumption, and thus (3.3) has a solution, since every $u_{i}^{(n)}$ and $\tilde{u}_{i}^{(n)}$ generated by the algorithm in (3.1) and (3.2) is attainable.

Proposition 3.1. The algorithms in (3.1) and (3.2) produce a sequence $\left(u^{(n)}\right)_{n}$ in $L^{2}(\Omega)$ with the following properties:

(i) $J_{\alpha_{1}, \alpha_{2}}\left(u^{(n)}\right) \geq J_{\alpha_{1}, \alpha_{2}}\left(u^{(n+1)}\right)$ for all $n \in \mathbb{N}$;

(ii) the sequence $\left(u^{(n)}\right)_{n}$ has subsequences that weakly converge in $L^{2}(\Omega)$ and $B V(\Omega)$. 
Proof. First we show (i) for the algorithm in (3.1). Observe that

$J_{\alpha_{1}, \alpha_{2}}\left(u^{(n)}\right)=J_{\alpha_{1}, \alpha_{2}}\left(\tilde{u}_{1}^{(n)}+\tilde{u}_{2}^{(n)}\right) \geq J_{\alpha_{1}, \alpha_{2}}\left(u_{1}^{(n+1)}+\tilde{u}_{2}^{(n)}\right) \geq J_{\alpha_{1}, \alpha_{2}}\left(u_{1}^{(n+1)}+u_{2}^{(n+1)}\right)=J_{\alpha_{1}, \alpha_{2}}\left(u^{(n+1)}\right)$,

which proves the assertion.

To show (i) for the algorithm in (3.2) we consider first that

$$
J_{\alpha_{1}, \alpha_{2}}\left(u^{(n)}\right) \geq \frac{1}{2}\left(J_{\alpha_{1}, \alpha_{2}}\left(u_{1}^{(n+1)}+\tilde{u}_{2}^{(n)}\right)+J_{\alpha_{1}, \alpha_{2}}\left(\tilde{u}_{1}^{(n)}+u_{2}^{(n+1)}\right)\right) .
$$

Moreover, by convexity we obtain

$$
J_{\alpha_{1}, \alpha_{2}}\left(\frac{u_{1}^{(n+1)}+u_{2}^{(n+1)}+\tilde{u}_{1}^{(n)}+\tilde{u}_{2}^{(n)}}{2}\right) \leq \frac{1}{2}\left(J_{\alpha_{1}, \alpha_{2}}\left(u_{1}^{(n+1)}+\tilde{u}_{2}^{(n)}\right)+J_{\alpha_{1}, \alpha_{2}}\left(\tilde{u}_{1}^{(n)}+u_{2}^{(n+1)}\right)\right)
$$

and hence $J_{\alpha_{1}, \alpha_{2}}\left(u^{(n)}\right) \geq J_{\alpha_{1}, \alpha_{2}}\left(u^{(n+1)}\right)$.

From the above considerations we infer that $J_{\alpha_{1}, \alpha_{2}}\left(u^{(0)}\right) \geq J_{\alpha_{1}, \alpha_{2}}\left(u^{(n)}\right)$ for all $n \in \mathbb{N}$. By the coercivity condition on $J_{\alpha_{1}, \alpha_{2}},\left(u^{(n)}\right)_{n}$ is uniformly bounded in $L^{2}(\Omega)$, and hence there exists a weakly convergent subsequence. Moreover, due to the presence of $\varphi(|D u|)$ in $J_{\alpha_{1}, \alpha_{2}}$ and $\alpha_{1}+\alpha_{2}>0$ we obtain that $\left(u^{(n)}\right)_{n}$ is bounded in $B V(\Omega)$. The compact embedding $B V(\Omega) \hookrightarrow L^{q}(\Omega), q<\frac{d}{d-1}$, implies that a subsequence $\left(u^{\left(n_{k}\right)}\right)_{k}$ converges in $L^{q}(\Omega)$ to a limit $u^{(\infty)} \in L^{2}(\Omega)$. By [3, Prop. 10.1.1] we even have that $u^{(\infty)} \in B V(\Omega)$, $\liminf _{n \rightarrow \infty} \varphi\left(\left|D u^{\left(n_{k}\right)}\right|\right)(\Omega) \geq \varphi\left(\left|D u^{(\infty)}\right|\right)(\Omega)$, and $u^{\left(n_{k}\right)}$ weakly converges to $u^{(\infty)}$ in $B V(\Omega)$, which concludes the proof.

Remark 3.2. Since the sequence $\left(J_{\alpha_{1}, \alpha_{2}}\left(u^{(n)}\right)\right)_{n}$ is monotonically decreasing and bounded from below, it is also convergent.

Proposition 3.3. The sequences $\left(u_{i}^{(n)}\right)_{n}$ and $\left(\tilde{u}_{i}^{(n)}\right)_{n}$ for $i=1,2$ generated by the algorithms in (3.1) or (3.2) are bounded in $L^{2}(\Omega)$ and hence have weak accumulation points $u_{i}^{(\infty)} \in L^{2}(\Omega)$ and $\tilde{u}_{i}^{(\infty)} \in L^{2}(\Omega)$, respectively.

Proof. The boundedness of $\left(u^{(n)}\right)_{n}$ implies the boundedness of $\left(\tilde{u}_{i}^{(n)}\right)_{n}$, since

$$
\left\|\tilde{u}_{i}^{(n)}\right\|_{L^{2}(\Omega)}=\left\|\chi_{i} u^{(n)}\right\|_{L^{2}(\Omega)} \leq \kappa\left\|u^{(n)}\right\|_{L^{2}(\Omega)} \leq C<\infty \quad \text { for } i=1,2,
$$

where $\kappa$ is defined as below (3.1). By the definition of $u_{1}^{(n+1)}$ and by the coercivity assumption on $J_{\alpha_{1}, \alpha_{2}}$ we have that $\left(u_{1}^{(n+1)}+\tilde{u}_{2}^{(n)}\right)_{n}$ is bounded in $L^{2}(\Omega)$; i.e., there exists a constant $C>0$ such that $\left\|u_{1}^{(n+1)}+\tilde{u}_{2}^{(n)}\right\|_{L^{2}(\Omega)} \leq C$ for all $n \in \mathbb{N}$. Since $\left(\tilde{u}_{2}^{(n)}\right)_{n}$ is bounded in $L^{2}(\Omega)$ by $(3.4)$, the triangle inequality yields

$$
\left\|u_{1}^{(n+1)}\right\|_{L^{2}(\Omega)}-\left\|\tilde{u}_{2}^{(n)}\right\|_{L^{2}(\Omega)} \leq\left\|u_{1}^{(n+1)}+\tilde{u}_{2}^{(n)}\right\|_{L^{2}(\Omega)} \leq C .
$$

Hence $\left(u_{1}^{(n)}\right)_{n}$ is bounded in $L^{2}(\Omega)$. By similar arguments we get the $L^{2}(\Omega)$-boundedness of $\left(u_{2}^{(n)}\right)_{n}$. Consequently $\left(\tilde{u}_{i}^{(n)}\right)_{n}$ and $\left(u_{i}^{(n)}\right)_{n}$ have a weakly convergent subsequence with limits $\tilde{u}_{i}^{(\infty)}$ and $u_{i}^{(\infty)}$, respectively, in $L^{2}(\Omega)$. 
4. Subspace correction approach for the discretized problem. In this section we analyze the method as it is implemented in finite dimensions upon discretization. We note, however, that a function space analysis appears possible as well, but would require careful handling of weak and weak-* convergent sequences as well as properties of the convex subdifferential.

4.1. Notation and basic definitions. In the rest of the paper we work on a finite regular mesh as a discretization of $\Omega$. We approximate functions $u$ by discrete functions, again denoted by $u$ with $\nabla u$ representing their gradient. Instead of the continuous functional (2.1) we consider its discrete approximation, for ease again denoted by $J_{\alpha_{1}, \alpha_{2}}$ in (4.1) below. Note that the discrete approximation $\Gamma$-converges to the continuous functional in $(2.1)$ (see $[7,37]$ ), and it has the same structural properties as the continuous one. Although in our applications we are mainly interested in imaging problems, i.e., two-dimensional problems, our notation covers any $d$-dimensional space.

In our discrete setting we define the discrete d-orthotope $\Omega=\left\{x_{1}^{1}<\cdots<x_{N_{1}}^{1}\right\} \times \cdots \times\left\{x_{1}^{d}<\right.$ $\left.\cdots<x_{N_{d}}^{d}\right\} \subset \mathbb{R}^{d}, d \in \mathbb{N}$, and the underlying "function space" is $\mathcal{H}=\mathbb{R}^{N_{1} \times N_{2} \times \cdots \times N_{d}}$, where $N_{j} \in \mathbb{N}$ for $j=1, \ldots, d$. Accordingly, $V_{1}$ and $V_{2}$ are appropriate subspaces of $\mathcal{H}$ such that $\mathcal{H}=V_{1}+V_{2}$, and by $V_{i}^{c}$ we denote the orthogonal complement of $V_{i}$ in $\mathcal{H}$ for $i=1,2$. For $u \in \mathcal{H}$ we write $u=u(x)=u\left(x_{i_{1}}^{1}, \ldots, x_{i_{d}}^{d}\right)$, where $i_{j} \in\left\{1, \ldots, N_{j}\right\}$ and $x \in \Omega$. Let $h=x_{i_{j}+1}^{j}-x_{i_{j}}^{j}$ be the equidistant step-size for all $j=1, \ldots, d$. We define the scalar products of $u, v \in \mathcal{H}$ and of $p, q \in \mathcal{H}^{d}$ by

$$
\langle u, v\rangle_{\mathcal{H}}=h^{d} \sum_{x \in \Omega} u(x) v(x) \quad \text { and } \quad\langle p, q\rangle_{\mathcal{H}^{d}}=h^{d} \sum_{x \in \Omega}\langle p(x), q(x)\rangle_{\mathbb{R}^{d}}
$$

with $\langle y, z\rangle_{\mathbb{R}^{d}}=\sum_{j=1}^{d} y_{j} z_{j}$ for every $y=\left(y_{1}, \ldots, y_{d}\right) \in \mathbb{R}^{d}$ and $z=\left(z_{1}, \ldots, z_{d}\right) \in \mathbb{R}^{d}$. In what follows we consider different norms. In particular we use

$$
\|u\|_{\ell^{p}(\Omega)}=\left(h^{d} \sum_{x \in \Omega}|u(x)|^{p}\right)^{1 / p}, \quad 1 \leq p<\infty,
$$

and $\|u\|_{\ell^{\infty}(\Omega)}=\sup _{x \in \Omega}|u(x)|$. Sometimes we do not specify the norm; i.e., we just write $\|\cdot\|$, which indicates that any norm can be taken.

The discrete gradient $\nabla u$ is denoted by $(\nabla u)(x)=\left((\nabla u)^{1}(x), \ldots,(\nabla u)^{d}(x)\right)$ with

$$
(\nabla u)^{j}(x)=\frac{1}{h} \cdot \begin{cases}u\left(x_{i_{1}}^{1}, \ldots, x_{i_{j}+1}^{j}, \ldots, x_{i_{d}}^{d}\right)-u\left(x_{i_{1}}^{1}, \ldots, x_{i_{j}}^{j}, \ldots, x_{i_{d}}^{d}\right) & \text { if } i_{j}<N_{j}, \\ 0 & \text { if } i_{j}=N_{j}\end{cases}
$$

for all $j=1, \ldots, d$ and for all $x \in \Omega$. Let $\varphi: \mathbb{R} \rightarrow \mathbb{R}$ with

$$
\varphi(|\omega|)(\Omega):=h^{d} \sum_{x \in \Omega} \varphi(|\omega(x)|)
$$

for $\omega \in \mathcal{H}^{d}$, where $|y|=\sqrt{y_{1}^{2}+\cdots+y_{d}^{2}}$. In particular we define the total variation of $u$ by setting $\varphi(t)=t$ and $\omega=\nabla u$; i.e.,

$$
|\nabla u|(\Omega):=h^{d} \sum_{x \in \Omega}|\nabla u(x)| .
$$


For an operator $T$ we denote by $T^{*}$ its adjoint. Further we introduce the discrete divergence $\operatorname{div}: \mathcal{H}^{d} \rightarrow \mathcal{H}$ defined by div $=-\nabla^{*}\left(\nabla^{*}\right.$ is the adjoint of the gradient $\left.\nabla\right)$, in analogy to the continuous setting. In our case, the discrete divergence operator is explicitly given by

$$
\begin{aligned}
(\operatorname{div} p)(x)= & \begin{cases}\frac{1}{h}\left(p^{1}\left(x_{i_{1}}^{1}, \ldots, x_{i_{d}}^{d}\right)-p^{1}\left(x_{i_{1}-1}^{1}, \ldots, x_{i_{d}}^{d}\right)\right) & \text { if } 1<i_{1}<N_{1}, \\
p^{1}\left(x_{i_{1}}^{1}, \ldots, x_{i_{d}}^{d}\right) & \text { if } i_{1}=1, \\
-p^{1}\left(x_{i_{1}-1}^{1}, \ldots, x_{i_{d}}^{d}\right) & \text { if } i_{1}=N_{1},\end{cases} \\
& +\cdots+ \begin{cases}\frac{1}{h}\left(p^{d}\left(x_{i_{1}}^{1}, \ldots, x_{i_{d}}^{d}\right)-p^{d}\left(x_{i_{1}}^{1}, \ldots, x_{i_{d}-1}^{d}\right)\right) & \text { if } 1<i_{d}<N_{d}, \\
p^{d}\left(x_{i_{1}}^{1}, \ldots, x_{i_{d}}^{d}\right) & \text { if } i_{d}=1, \\
-p^{d}\left(x_{i_{1}}^{1}, \ldots, x_{i_{d}-1}^{d}\right) & \text { if } i_{d}=N_{d}\end{cases}
\end{aligned}
$$

for every $p=\left(p^{1}, \ldots, p^{d}\right) \in \mathcal{H}^{d}$ and for all $x \in \Omega$. (Note that if the discrete domains $\Omega$ are not discrete $d$-orthotopes, then the definitions of the gradient and divergence operators have to be adjusted accordingly.) We will often use the symbol 1 to indicate the constant vector with entry values 1 , and $1_{D}$ to indicate the characteristic function of the domain $D \subset \Omega$.

For a convex functional $\mathcal{J}: \mathcal{H} \rightarrow \overline{\mathbb{R}}$, we define the subdifferential of $\mathcal{J}$ at $v \in \mathcal{H}$ as the set valued mapping

$$
\partial \mathcal{J}(v):= \begin{cases}\emptyset & \text { if } \mathcal{J}(v)=\infty, \\ \left\{v^{*} \in \mathcal{H}:\left\langle v^{*}, u-v\right\rangle_{\mathcal{H}}+\mathcal{J}(v) \leq \mathcal{J}(u) \quad \forall u \in \mathcal{H}\right\} & \text { otherwise }\end{cases}
$$

at $v \in \mathcal{H}$. It is clear from this definition that $0 \in \partial \mathcal{J}(v)$ if and only if $v$ is a minimizer of $\mathcal{J}$. Since we consider different spaces, namely $\mathcal{H}, V_{i}$, it is sometimes useful to indicate this in the notation of the subdifferential; e.g., we write $\partial_{V_{i}} \mathcal{J}$ whenever the subdifferential of $\mathcal{J}$ is taken with respect to $V_{i}$.

4.2. Properties of subspace correction methods. In what follows we consider the discrete functional

$$
J_{\alpha_{1}, \alpha_{2}}(u)=\alpha_{1}\left\|T_{1} u-g_{1}\right\|_{\ell^{1}(\Omega)}+\alpha_{2}\left\|T_{2} u-g_{2}\right\|_{\ell^{2}(\Omega)}^{2}+\varphi(|\nabla u|)(\Omega),
$$

where $T_{i}: \mathcal{H} \rightarrow \mathcal{H}$ is a bounded linear operator, $g_{i} \in \mathcal{H}$ is a given datum, and $\alpha_{i} \geq 0$ for $i=1,2$ with $\alpha_{1}+\alpha_{2}>0$. Moreover, we assume that $\varphi$ fulfills assumption $\left(A_{\varphi}\right)$ and that $J_{\alpha_{1}, \alpha_{2}}$ is bounded from below and coercive.

In the sequential and parallel algorithms in (3.1) and (3.2) we denote the difference between the current subspace minimizer $u_{i}^{(n+1)}$ and the initial value $\tilde{u}_{i}^{(n)}$ by $s^{\left(n+\frac{i}{2}\right)}$; i.e.,

$$
s^{\left(n+\frac{i}{2}\right)}:=u_{i}^{(n+1)}-\tilde{u}_{i}^{(n)} \text { for } i=1,2 .
$$

It is easy to see that in the sequential domain decomposition algorithm

$$
s^{\left(n+\frac{i}{2}\right)}=\arg \min _{s} J_{\alpha_{1}, \alpha_{2}}\left(u^{(n)}+(i-1) s^{\left(n+\frac{i-1}{2}\right)}+s\right) \quad \text { s.t. } \pi_{V_{i}^{c}} s=0,
$$

and in the parallel version

$$
s^{\left(n+\frac{i}{2}\right)}=\arg \min _{s} J_{\alpha_{1}, \alpha_{2}}\left(u^{(n)}+s\right) \quad \text { s.t. } \pi_{V_{i}^{c}} s=0
$$


for $i=1,2$. Moreover, for $v, s \in \mathcal{H}$ a quadratic Taylor expansion yields

$$
\begin{aligned}
\min _{s} J_{\alpha_{1}, \alpha_{2}}(v+s)= & \min _{s} 2 \alpha_{2}\left\langle T_{2} s, T_{2} v-g_{2}\right\rangle_{\mathcal{H}}+\alpha_{2}\left\|T_{2} s\right\|_{\ell^{2}(\Omega)}^{2} \\
& +\alpha_{1}\left\|T_{1}(v+s)-g_{1}\right\|_{\ell^{1}(\Omega)}+\varphi(|\nabla(v+s)|)(\Omega) .
\end{aligned}
$$

Then the following lemma can be proven similarly to Lemma 1 of [49]. For its statement we define the quantities $v(i), i=1,2$, as follows: For the sequential domain decomposition algorithm in (3.1) choose $v(i)=u^{(n)}$ if $i=1$ and $v(i)=u^{(n)}+s^{\left(n+\frac{1}{2}\right)}$ for $i=2$, while for the parallel domain decomposition algorithm in (3.2), $v(i)=u^{(n)}$ for $i=1,2$.

Lemma 4.1. Let $P(u)=\alpha_{1}\left\|T_{1} u-g_{1}\right\|_{\ell^{1}(\Omega)}+\varphi(|\nabla u|)(\Omega)$. For any $v(i) \in \mathcal{H}$ chosen according to the underlying algorithm, let $\tilde{s}=s^{\left(n+\frac{i}{2}\right)}$ for $i=1,2$. Then

$J_{\alpha_{1}, \alpha_{2}}(v(i)+\tilde{s})=J_{\alpha_{1}, \alpha_{2}}(v(i))+2 \alpha_{2}\left\langle T_{2} \tilde{s}, T_{2} v(i)-g_{2}\right\rangle_{\mathcal{H}}+\alpha_{2}\left\|T_{2} \tilde{s}\right\|_{\ell^{2}(\Omega)}^{2}+P(v(i)+\tilde{s})-P(v(i))$

and

$$
2 \alpha_{2}\left\langle T_{2} \tilde{s}, T_{2} v(i)-g_{2}\right\rangle_{\mathcal{H}}+P(v(i)+\tilde{s})-P(v(i)) \leq-2 \alpha_{2}\left\|T_{2} \tilde{s}\right\|_{\ell^{2}(\Omega)}^{2} .
$$

Remark 4.2. With $\alpha_{2}>0, v(i)$ as above and $\tilde{s}=s^{\left(n+\frac{i}{2}\right)}$, a direct consequence of Lemma 4.1 is that

$$
J_{\alpha_{1}, \alpha_{2}}(v(i)+\tilde{s})-J_{\alpha_{1}, \alpha_{2}}(v(i)) \leq-\alpha_{2}\left\|T_{2} \tilde{s}\right\|_{\ell^{2}(\Omega)}^{2},
$$

where $\alpha_{2}\left\|T_{2} \tilde{s}\right\|_{\ell^{2}(\Omega)}^{2}>0$ whenever $\tilde{s} \notin \operatorname{ker} T_{2}$. Note that the above descent property holds in particular when $T_{2}^{*} T_{2}$ is invertible and $\|\tilde{s}\| \neq 0$.

Proposition 4.3. Assume that $T_{2}^{*} T_{2}$ is invertible and $\alpha_{2}>0$. Let the sequence $\left(u^{(n)}\right)_{n}$ be generated by the algorithm in (3.1) or (3.2), and let $s^{\left(n+\frac{i}{2}\right)}$ be defined as in (4.2). Then we have the following statements:

(i) $\left\|s^{\left(n+\frac{i}{2}\right)}\right\| \rightarrow 0$ for $n \rightarrow \infty$,

(ii) $\left\|u^{(n+1)}-u^{(n)}\right\| \rightarrow 0$ for $n \rightarrow \infty$.

Proof. We begin by showing these statements for the sequential algorithm in (3.1). By the minimality property of $s^{\left(n+\frac{i}{2}\right)}$ we have that whenever $\left\|s^{\left(n+\frac{i}{2}\right)}\right\| \neq 0$,

$$
J_{\alpha_{1}, \alpha_{2}}\left(u^{(n)}+(i-1) s^{\left(n+\frac{i-1}{2}\right)}+s^{\left(n+\frac{i}{2}\right)}\right)<J_{\alpha_{1}, \alpha_{2}}\left(u^{(n)}+(i-1) s^{\left(n+\frac{i-1}{2}\right)}\right)
$$

for $i=1,2$. Note that $u^{(n+1)}=u^{(n)}+s^{\left(n+\frac{1}{2}\right)}+s^{(n+1)}$. Analogously to Remark 3.2, we find that $J_{\alpha_{1}, \alpha_{2}}$ is convergent, and hence by the above observation we obtain

$$
J_{\alpha_{1}, \alpha_{2}}\left(u^{(n)}+(i-1) s^{\left(n+\frac{i-1}{2}\right)}+s^{\left(n+\frac{i}{2}\right)}\right)-J_{\alpha_{1}, \alpha_{2}}\left(u^{(n)}+(i-1) s^{\left(n+\frac{i-1}{2}\right)}\right) \rightarrow 0 \text { for } n \rightarrow \infty .
$$

By (4.3) it follows then that $\left\|s^{\left(n+\frac{i}{2}\right)}\right\| \rightarrow 0$ for $n \rightarrow \infty$ and for $i=1,2$, which proves (i). Since $u^{(n+1)}=u^{(n)}+s^{\left(n+\frac{1}{2}\right)}+s^{(n+1)}$, (ii) immediately follows.

For the parallel algorithm in (3.2) we obtain by the minimality property of $s^{\left(n+\frac{i}{2}\right)}$ that

$$
J_{\alpha_{1}, \alpha_{2}}\left(u^{(n)}+s^{\left(n+\frac{i}{2}\right)}\right)<J_{\alpha_{1}, \alpha_{2}}\left(u^{(n)}\right)
$$


for $i=1,2$ whenever $\left\|s^{\left(n+\frac{i}{2}\right)}\right\| \neq 0$. Hence by convexity and the definition of $u^{(n+1)}$ in $(3.2)$ we get

$$
2 J_{\alpha_{1}, \alpha_{2}}\left(u^{(n)}\right)>J_{\alpha_{1}, \alpha_{2}}\left(u^{(n)}+s^{\left(n+\frac{1}{2}\right)}\right)+J_{\alpha_{1}, \alpha_{2}}\left(u^{(n)}+s^{(n+1)}\right) \geq 2 J_{\alpha_{1}, \alpha_{2}}\left(u^{(n+1)}\right) .
$$

From (4.4), the convergence of $J_{\alpha_{1}, \alpha_{2}}$, and the previous inequalities we obtain

$$
\begin{aligned}
& \underbrace{J_{\alpha_{1}, \alpha_{2}}\left(u^{(n)}\right)-J_{\alpha_{1}, \alpha_{2}}\left(u^{(n)}+s^{\left(n+\frac{1}{2}\right)}\right)}_{\geq 0}+\underbrace{J_{\alpha_{1}, \alpha_{2}}\left(u^{(n)}\right)-J_{\alpha_{1}, \alpha_{2}}\left(u^{(n)}+s^{(n+1)}\right)}_{\geq 0} \\
& \quad \leq 2\left(J_{\alpha_{1}, \alpha_{2}}\left(u^{(n)}\right)-J_{\alpha_{1}, \alpha_{2}}\left(u^{(n+1)}\right)\right) \rightarrow 0 \text { for } n \rightarrow \infty .
\end{aligned}
$$

By (4.3) we eventually have that $\left\|s^{\left(n+\frac{i}{2}\right)}\right\| \rightarrow 0$ for $n \rightarrow \infty$ and for $i=1,2$. The second statement follows from $u^{(n+1)}=\frac{u_{1}^{(n+1)}+u_{2}^{(n+1)}+u^{(n)}}{2}$, since

$$
\left\|u^{(n+1)}-u^{(n)}\right\|=\left\|\frac{u_{1}^{(n+1)}+u_{2}^{(n+1)}-u^{(n)}}{2}\right\|=\left\|\frac{s^{\left(n+\frac{1}{2}\right)}+s^{(n+1)}}{2}\right\| .
$$

Remark 4.4. From the proof of Proposition 4.3 we find that if $T_{2}^{*} T_{2}$ is invertible and $\alpha_{2}>0$, then we can replace the nonincrease of the energy $J_{\alpha_{1}, \alpha_{2}}$ in Proposition 3.1(i) by a strict monotone decrease unless $u^{(n+1)}=u^{(n)}$.

4.2.1. A convergence estimate. For proving convergence results of the algorithms in (3.1) and (3.2) we use a characterization of solutions of the minimization problem (2.1) similar to [50, Prop. 4.1] in the continuous setting for $\alpha_{1}=0$ and adapted in [29, Prop. 5.2] to the discrete case.

Characterization of solutions. Following [26, Def. 4.1, p. 17] the conjugate (or Legendre transform) of a convex function $\phi: V \rightarrow \mathbb{R}$, with $V$ a vector space with topological dual $V^{*}$ and duality pairing $\langle\cdot, \cdot\rangle$, is defined by

$$
\phi^{*}\left(u^{*}\right)=\sup _{u \in V}\left\{\left\langle u, u^{*}\right\rangle-\phi(u)\right\} .
$$

The convex conjugate is useful when characterizing the solution to (4.1) with $\alpha_{1}, \alpha_{2}>0$.

Proposition 4.5. Let $\zeta, u \in \mathcal{H}$. If assumption $\left(A_{\varphi}\right)$ holds true, then $\zeta \in \partial J_{\alpha_{1}, \alpha_{2}}(u)$ if and only if there exist $M=\left(M_{0}, M_{1}, M_{2}\right) \in \mathcal{H}^{d} \times \mathcal{H} \times \mathcal{H}$ and a constant $c_{1} \geq 0$ with $\left|M_{0}(x)\right| \leq c_{1}$, $\left|M_{1}(x)\right| \leq \alpha_{1}$ for all $x \in \Omega$ such that

$$
\begin{array}{rr}
\varphi(|(\nabla u)(x)|)+\left\langle M_{0}(x), \nabla u(x)\right\rangle_{\mathbb{R}^{d}}+\varphi_{1}^{*}\left(\left|M_{0}(x)\right|\right)=0 & \forall x \in \Omega, \\
M_{2}(x)=-2 \alpha_{2}\left(T_{2} u-g_{2}\right)(x) & \forall x \in \Omega, \\
\alpha_{1}\left|\left(T_{1} u-g_{1}\right)(x)\right|+M_{1}(x)\left(\left(T_{1} u\right)(x)-g_{1}(x)\right)=0 & \forall x \in \Omega, \\
T_{1}^{*} M_{1}+T_{2}^{*} M_{2}-\operatorname{div} M_{0}+\zeta=0,
\end{array}
$$

where $\varphi_{1}^{*}$ is the conjugate function of $\varphi_{1}$ defined by $\varphi_{1}(t)=\varphi(|t|)$ for $t \in \mathbb{R}$. If, additionally, $\varphi$ is differentiable and $|(\nabla u)(x)| \neq 0$ for $x \in \Omega$, then we can compute $M_{0}$ as

$$
M_{0}(x)=-\frac{\varphi^{\prime}(|(\nabla u)(x)|)}{|(\nabla u)(x)|}(\nabla u)(x) .
$$


The proof of this proposition is deferred to the appendix.

For $\alpha_{2}=0$ the minimization problem associated with the objective in (4.1) becomes

$$
\min _{u \in \mathcal{H}} J_{\alpha_{1}, 0}(u)=\alpha_{1}\left\|\left(T_{1} u-g_{1}\right)\right\|_{\ell^{1}(\Omega)}+\varphi(|\nabla u|)(\Omega),
$$

and system (4.5)-(4.8) reduces to (4.11)-(4.13) below.

Corollary 4.6. Let $\zeta, u \in \mathcal{H}$. If assumption $\left(A_{\varphi}\right)$ holds true, then $\zeta \in \partial J_{\alpha_{1}, 0}(u)$ if and only if there exist $M=\left(M_{0}, M_{1}\right) \in \mathcal{H}^{d} \times \mathcal{H}$ and a constant $c_{1} \geq 0$ with $\left|M_{0}(x)\right| \leq c_{1},\left|M_{1}(x)\right| \leq \alpha_{1}$ for all $x \in \Omega$ such that

$$
\begin{array}{r}
\varphi(|(\nabla u)(x)|)+\left\langle M_{0}(x), \nabla u(x)\right\rangle_{\mathbb{R}^{d}}+\varphi_{1}^{*}\left(\left|M_{0}(x)\right|\right)=0 \quad \forall x \in \Omega, \\
\alpha_{1}\left|\left(T_{1} u-g_{1}\right)(x)\right|+M_{1}(x)\left(\left(T_{1} u\right)(x)-g_{1}(x)\right)=0 \quad \forall x \in \Omega, \\
T_{1}^{*} M_{1}-\operatorname{div} M_{0}+\zeta=0,
\end{array}
$$

where $\varphi_{1}^{*}$ is the conjugate function of $\varphi_{1}$ defined by $\varphi_{1}(t)=\varphi(|t|)$ for $t \in \mathbb{R}$.

Optimality properties. By [35, Thm. 2.1.4, p. 305], the optimality condition for the subspace minimization problem in $V_{i}$ (cf. (3.3)), i.e.,

$$
\xi_{i}^{(n+1)} \in \arg \min _{\xi_{i} \in \mathcal{H}}\left\{J_{\alpha_{1}, \alpha_{2}}\left(\xi_{i}\right): \pi_{V_{i}^{c}} \xi_{i}=\pi_{V_{i}^{c}} b\right\},
$$

is

$$
0 \in \partial J_{\alpha_{1}, \alpha_{2}}\left(\xi_{i}^{(n+1)}\right)+\eta_{i}^{(n+1)},
$$

where $\eta_{i}^{(n+1)} \in \operatorname{Range}\left(\pi_{V_{i}^{c}}^{*}\right) \simeq V_{i}^{c}$ and $b \in V_{j}$ as in (3.3) for $i=1,2$ and $j \in\{1,2\} \backslash\{i\}$. Note that indeed $\xi_{1}^{(n+1)}$ is optimal for (4.14) with $i=1$ and $b=\tilde{u}_{2}^{(n)}$ if and only if $u_{1}^{(n+1)}=\xi_{1}^{(n+1)}$ $\tilde{u}_{2}^{(n)}$ is optimal for the first minimization problem in (3.1) or (3.2), respectively. Moreover, $\xi_{2}^{(n+1)}$ is optimal for (4.14) with $i=2$ and $b=u_{1}^{(n+1)}$ if and only if $u_{2}^{(n+1)}=\xi_{2}^{(n+1)}-u_{1}^{(n+1)}$ is a solution of the second minimization problem in (3.1), and $\xi_{2}^{(n+1)}$ is optimal for (4.14) with $i=2$ and $b=\tilde{u}_{1}^{(n)}$ if and only if $u_{2}^{(n+1)}=\xi_{2}^{(n+1)}-\tilde{u}_{1}^{(n)}$ is optimal for the second minimization problem in (3.2).

The following result is a consequence of Proposition 4.5 and Corollary 4.6. It relies on the fact that $\partial J_{\alpha_{1}, \alpha_{2}}(\xi)$ is compact for any $\xi \in \mathcal{H}$; see [6].

Corollary 4.7. Let $\left(\xi^{(n)}\right)_{n} \subset \mathcal{H}$ be bounded and $\eta^{(n)} \in \partial J_{\alpha_{1}, \alpha_{2}}\left(\xi^{(n)}\right)$ for all $n \in \mathbb{N}$. Then $\left(\eta^{(n)}\right)_{n}$ is bounded.

Proof. Set $P\left(\xi^{(n)}\right):=\alpha_{1}\left\|T_{1} \xi^{(n)}-g_{1}\right\|_{\ell^{1}(\Omega)}+\varphi\left(\left|\nabla \xi^{(n)}\right|\right)(\Omega)$. Then we have

$$
\eta^{(n)} \in 2 \alpha_{2} T_{2}^{*}\left(T_{2} \xi^{(n)}-g_{2}\right)+\partial P\left(\xi^{(n)}\right) .
$$

Since $T_{2}$ is a bounded operator and $\left(\xi^{(n)}\right)_{n}$ is bounded, we are left with showing that the set $\partial P\left(\xi^{(n)}\right)$ is bounded for all $n$. By Corollary 4.6 we have that

$$
\begin{aligned}
\partial P\left(\xi^{(n)}\right)=\{ & \operatorname{div} M_{0}-T_{1}^{*} M_{1} \in \mathcal{H}:\left\|M_{0}\right\|_{\infty} \leq c_{1},\left\|M_{1}\right\|_{\infty} \leq \alpha_{1}, \\
& \varphi\left(\left|\left(\nabla \xi^{(n)}\right)(x)\right|\right)+\left\langle M_{0}(x), \nabla \xi^{(n)}(x)\right\rangle_{\mathbb{R}^{d}}+\varphi_{1}^{*}\left(\left|M_{0}(x)\right|\right)=0, \\
& \left.\alpha_{1}\left|\left(T_{1} \xi^{(n)}-g_{1}\right)(x)\right|+M_{1}(x)\left(\left(T_{1} \xi^{(n)}\right)(x)-g_{1}(x)\right)=0 \forall x \in \Omega\right\} .
\end{aligned}
$$


Since $c_{1}$ and $\alpha_{1}$ do not depend on $n$, the sequence of sets $\left(\partial P\left(\xi^{(n)}\right)\right)_{n}$ is uniformly bounded, and hence $\left(\eta^{(n)}\right)_{n}$ is bounded as well.

Proposition 4.8. There exist accumulation points $u_{i}^{(\infty)}$ and $\tilde{u}_{i}^{(\infty)}$ of the sequences $\left(u_{i}^{(n)}\right)_{n}$ and $\left(\tilde{u}_{i}^{(n)}\right)_{n}, i=1,2$, generated by the algorithms in (3.1) or (3.2) such that

(i) $u_{1}^{(\infty)}$ and $\tilde{u}_{1}^{(\infty)}$ are minimizers of $\min _{u_{1} \in V_{1}} J_{\alpha_{1}, \alpha_{2}}\left(u_{1}+\tilde{u}_{2}^{(\infty)}\right)$ and

(ii) for the algorithm in (3.1), $u_{2}^{(\infty)}$ and $\tilde{u}_{2}^{(\infty)}$ are minimizers of $\min _{u_{2} \in V_{2}} J_{\alpha_{1}, \alpha_{2}}\left(u_{1}^{(\infty)}+u_{2}\right)$, and for the algorithm in $(3.2), u_{2}^{(\infty)}$ and $\tilde{u}_{2}^{(\infty)}$ are minimizers of $\min _{u_{2} \in V_{2}} J_{\alpha_{1}, \alpha_{2}}\left(\tilde{u}_{1}^{(\infty)}+\right.$ $\left.u_{2}\right)$.

Proof. We start by showing the assertion for algorithm (3.1). By Proposition 3.3 the sequence $\left(\tilde{u}_{2}^{(n)}\right)_{n}$ is bounded and hence has a convergent subsequence $\left(\tilde{u}_{2}^{\left(n_{k}\right)}\right)_{k}$ with limit $\tilde{u}_{2}^{(\infty)}$. Further, $\left(u_{1}^{\left(n_{k}+1\right)}\right)_{k}$ is bounded and has a subsequence $\left(u_{1}^{\left(n_{k_{\ell}}+1\right)}\right)_{\ell}$ which converges to $u_{1}^{(\infty)}$. The minimality property of $u_{1}^{\left(n_{k_{\ell}}+1\right)}$ yields that $0 \in \partial_{V_{1}} J_{\alpha_{1}, \alpha_{2}}\left(\cdot+\tilde{u}_{2}^{\left(n_{k_{\ell}}\right)}\right)\left(u_{1}^{\left(n_{k_{\ell}}+1\right)}\right)$. By [44, Thm. 24.4, p. 233] we obtain that $0 \in \partial_{V_{1}} J_{\alpha_{1}, \alpha_{2}}\left(\cdot+\tilde{u}_{2}^{(\infty)}\right)\left(u_{1}^{(\infty)}\right)$ and hence $u_{1}^{(\infty)} \in$ $\arg \min _{u_{1} \in V_{1}} J_{\alpha_{1}, \alpha_{2}}\left(u_{1}+\tilde{u}_{2}^{(\infty)}\right)$. Since $\left(u_{2}^{\left(n_{k_{\ell}}+1\right)}\right)_{\ell}$ is again bounded, there exists a convergent subsequence $\left(u_{2}^{\left(n_{k_{\ell_{j}}}+1\right)}\right)_{j}$ with limit $u_{2}^{(\infty)}$, and we get that $0 \in \partial_{V_{2}} J_{\alpha_{1}, \alpha_{2}}\left(u_{1}^{(\infty)}+\cdot\right)\left(u_{2}^{(\infty)}\right)$, i.e., $u_{2}^{(\infty)}=\arg \min _{u_{2} \in V_{2}} J_{\alpha_{1}, \alpha_{2}}\left(u_{1}^{(\infty)}+u_{2}\right)$. Moreover, by the monotone decrease of the energy (see Proposition 3.1), we have that

$$
J_{\alpha_{1}, \alpha_{2}}\left(u^{(n)}\right) \geq J_{\alpha_{1}, \alpha_{2}}\left(u_{1}^{(n+1)}+\tilde{u}_{2}^{(n)}\right) \geq J_{\alpha_{1}, \alpha_{2}}\left(u^{(n+1)}\right) \quad \forall n \in \mathbb{N},
$$

and hence

$$
J_{\alpha_{1}, \alpha_{2}}\left(u^{(n)}\right)-J_{\alpha_{1}, \alpha_{2}}\left(u^{(n+1)}\right) \geq J_{\alpha_{1}, \alpha_{2}}\left(u_{1}^{(n+1)}+\tilde{u}_{2}^{(n)}\right)-J_{\alpha_{1}, \alpha_{2}}\left(\tilde{u}_{1}^{(n+1)}+\tilde{u}_{2}^{(n+1)}\right) \geq 0
$$

as well as

$$
J_{\alpha_{1}, \alpha_{2}}\left(u^{(n)}\right)-J_{\alpha_{1}, \alpha_{2}}\left(u^{(n+1)}\right) \geq J_{\alpha_{1}, \alpha_{2}}\left(u_{1}^{(n+1)}+\tilde{u}_{2}^{(n)}\right)-J_{\alpha_{1}, \alpha_{2}}\left(u_{1}^{(n+1)}+u_{2}^{(n+1)}\right) \geq 0 .
$$

Since $J_{\alpha_{1}, \alpha_{2}}$ is bounded from below, we obtain $\lim _{n \rightarrow \infty}\left[J_{\alpha_{1}, \alpha_{2}}\left(u^{(n)}\right)-J_{\alpha_{1}, \alpha_{2}}\left(u^{(n+l)}\right)\right]=0$ for any $l \in \mathbb{N}$. Consequently

$$
\begin{aligned}
& 0=\lim _{n_{k_{\ell}} \rightarrow \infty}\left[J_{\alpha_{1}, \alpha_{2}}\left(u_{1}^{\left(n_{k_{\ell}}+1\right)}+\tilde{u}_{2}^{\left(n_{k_{\ell}}\right)}\right)-J_{\alpha_{1}, \alpha_{2}}\left(\tilde{u}_{1}^{\left(n_{k_{\ell+1}}\right)}+\tilde{u}_{2}^{\left(n_{k_{\ell+1}}\right)}\right)\right], \\
& 0=\lim _{n_{k_{\ell_{j}} \rightarrow \infty}}\left[J_{\alpha_{1}, \alpha_{2}}\left(u_{1}^{\left(n_{k_{\ell_{j}}}+1\right)}+\tilde{u}_{2}^{\left(n_{k_{\ell_{j}}}\right)}\right)-J_{\alpha_{1}, \alpha_{2}}\left(u_{1}^{\left(n_{k_{\ell_{j}}}+1\right)}+u_{2}^{\left(n_{k_{\ell_{j}}}+1\right)}\right)\right] \\
& =J_{\alpha_{1}, \alpha_{2}}\left(u_{1}^{(\infty)}+\tilde{u}_{2}^{(\infty)}\right)-J_{\alpha_{1}, \alpha_{2}}\left(u_{1}^{(\infty)}+u_{2}^{(\infty)}\right) .
\end{aligned}
$$

From (4.17) we observe that since $u_{2}^{(\infty)} \in \arg \min _{u_{2} \in V_{2}} J_{\alpha_{1}, \alpha_{2}}\left(u_{1}^{(\infty)}+u_{2}\right)$, then also $\tilde{u}_{2}^{(\infty)} \in$ $\arg \min _{u_{2} \in V_{2}} J_{\alpha_{1}, \alpha_{2}}\left(u_{1}^{(\infty)}+u_{2}\right)$. 
Since $\left(\tilde{u}_{1}^{\left(n_{k_{\ell+1}}\right)}\right)_{\ell}$ is bounded, there exists a convergent subsequence $\left(\tilde{u}_{1}^{\left(n_{k_{\ell}+1}\right)}\right)_{m}$ with limit $\tilde{u}_{1}^{(\infty)}$, and we obtain from $(4.16)$

$$
\begin{aligned}
0 & =\lim _{n_{k_{m}} \rightarrow \infty}\left[J_{\alpha_{1}, \alpha_{2}}\left(u_{1}^{\left(n_{k_{\ell_{m}}}+1\right)}+\tilde{u}_{2}^{\left(n_{k_{\ell_{m}}}\right)}\right)-J_{\alpha_{1}, \alpha_{2}}\left(\tilde{u}_{1}^{\left(n_{k_{\ell_{m}+1}}\right)}+\tilde{u}_{2}^{\left(n_{k_{\ell_{m}+1}}\right)}\right)\right] \\
& =J_{\alpha_{1}, \alpha_{2}}\left(u_{1}^{(\infty)}+\tilde{u}_{2}^{(\infty)}\right)-J_{\alpha_{1}, \alpha_{2}}\left(\tilde{u}_{1}^{(\infty)}+\tilde{u}_{2}^{(\infty)}\right) .
\end{aligned}
$$

From (4.18) we infer that since $u_{1}^{(\infty)}$ is a solution of $\min _{u_{1} \in V_{1}} J_{\alpha_{1}, \alpha_{2}}\left(u_{1}+\tilde{u}_{2}^{(\infty)}\right)$, $\tilde{u}_{1}^{(\infty)}$ is a solution as well.

Although the proof for the algorithm in (3.2) is similar, for the sake of completeness we provide the details here. By similar arguments as above, one shows that there exist accumulation points $u_{1}^{(\infty)} \in \arg \min _{u_{1} \in V_{1}} J_{\alpha_{1}, \alpha_{2}}\left(u_{1}+\tilde{u}_{2}^{(\infty)}\right)$ and $u_{2}^{(\infty)}=\arg \min _{u_{2} \in V_{2}} J_{\alpha_{1}, \alpha_{2}}\left(\tilde{u}_{1}^{(\infty)}+u_{2}\right)$. Hence, we have

$$
\begin{array}{ll}
J_{\alpha_{1}, \alpha_{2}}\left(u_{1}^{(\infty)}+\tilde{u}_{2}^{(\infty)}\right) \leq J_{\alpha_{1}, \alpha_{2}}\left(u_{1}+\tilde{u}_{2}^{(\infty)}\right) & \forall u_{1} \in V_{1}, \\
J_{\alpha_{1}, \alpha_{2}}\left(\tilde{u}_{1}^{(\infty)}+u_{2}^{(\infty)}\right) \leq J_{\alpha_{1}, \alpha_{2}}\left(\tilde{u}_{1}^{(\infty)}+u_{2}\right) & \forall u_{2} \in V_{2} .
\end{array}
$$

Moreover, by the monotone decrease of the energy $J_{\alpha_{1}, \alpha_{2}}$ (see Proposition 3.1), we have

$$
J_{\alpha_{1}, \alpha_{2}}\left(u^{(n)}\right) \geq \frac{1}{2}\left(J_{\alpha_{1}, \alpha_{2}}\left(u_{1}^{(n+1)}+\tilde{u}_{2}^{(n)}\right)+J_{\alpha_{1}, \alpha_{2}}\left(\tilde{u}_{1}^{(n)}+u_{2}^{(n+1)}\right)\right) \geq J_{\alpha_{1}, \alpha_{2}}\left(u^{(n+1)}\right)
$$

and hence

$$
\begin{aligned}
& 2\left(J_{\alpha_{1}, \alpha_{2}}\left(u^{(n)}\right)-J_{\alpha_{1}, \alpha_{2}}\left(u^{(n+1)}\right)\right) \\
& \quad \geq J_{\alpha_{1}, \alpha_{2}}\left(u_{1}^{(n+1)}+\tilde{u}_{2}^{(n)}\right)+J_{\alpha_{1}, \alpha_{2}}\left(\tilde{u}_{1}^{(n)}+u_{2}^{(n+1)}\right)-2 J_{\alpha_{1}, \alpha_{2}}\left(u^{(n+1)}\right) \geq 0 .
\end{aligned}
$$

Since $J_{\alpha_{1}, \alpha_{2}}$ is bounded below, we obtain $\lim _{n \rightarrow \infty}\left[J_{\alpha_{1}, \alpha_{2}}\left(u^{(n)}\right)-J_{\alpha_{1}, \alpha_{2}}\left(u^{(n+l)}\right)\right]=0$ for any $l \in \mathbb{N}$, and hence

$$
\begin{array}{r}
\lim _{n \rightarrow \infty}\left[J_{\alpha_{1}, \alpha_{2}}\left(u_{1}^{\left(n_{k_{\ell_{m}}}+1\right)}+\tilde{u}_{2}^{\left(n_{k_{\ell_{j}}}\right)}\right)+J_{\alpha_{1}, \alpha_{2}}\left(\tilde{u}_{1}^{\left(n_{k_{\ell_{j}}}\right)}+u_{2}^{\left(n_{k_{\ell_{j}}}+1\right)}\right)\right. \\
\left.-2 J_{\alpha_{1}, \alpha_{2}}\left(\tilde{u}_{1}^{\left(n_{k_{\ell_{m}+1}}\right)}+\tilde{u}_{2}^{\left(n_{k_{\ell_{m}+1}}\right)}\right)\right]=0 .
\end{array}
$$

From (4.19) it follows that

$J_{\alpha_{1}, \alpha_{2}}\left(u_{1}^{(\infty)}+\tilde{u}_{2}^{(\infty)}\right)-J_{\alpha_{1}, \alpha_{2}}\left(\tilde{u}_{1}^{(\infty)}+\tilde{u}_{2}^{(\infty)}\right)=0 \quad$ and $\quad J_{\alpha_{1}, \alpha_{2}}\left(\tilde{u}_{1}^{(\infty)}+u_{2}^{(\infty)}\right)-J_{\alpha_{1}, \alpha_{2}}\left(\tilde{u}_{1}^{(\infty)}+\tilde{u}_{2}^{(\infty)}\right)=0$,

and hence

$$
\tilde{u}_{1}^{(\infty)} \in \arg \min _{u_{1} \in V_{1}} J_{\alpha_{1}, \alpha_{2}}\left(u_{1}+\tilde{u}_{2}^{(\infty)}\right) \quad \text { and } \quad \tilde{u}_{2}^{(\infty)} \in \arg \min _{u_{2} \in V_{2}} J_{\alpha_{1}, \alpha_{2}}\left(\tilde{u}_{1}^{(\infty)}+u_{2}\right),
$$

which concludes the proof. 


\section{Remark 4.9.}

(i) If $V_{1}$ and $V_{2}$ are disjoint spaces, then the algorithm in (3.1) generates sequences $\left(u_{i}^{(n)}\right)_{n}$ and $\left(\tilde{u}_{i}^{(n)}\right)_{n}$ with $u_{i}^{(n)}=\tilde{u}_{i}^{(n)}$, and the algorithm in (3.2) generates sequences $\left(u_{i}^{(n)}\right)_{n}$ and $\left(\tilde{u}_{i}^{(n)}\right)_{n}$ with $\tilde{u}_{i}^{(n+1)}=\frac{1}{2}\left(u_{i}^{(n+1)}+u_{i}^{(n)}\right), \tilde{u}_{i}^{(\infty)}=u_{i}^{(\infty)}$, and $u^{(\infty)}=u_{1}^{(\infty)}+u_{2}^{(\infty)}$.

(ii) In general, however, the algorithms in (3.1) and (3.2), respectively, may generate sequences $\left(u_{i}^{(n)}\right)_{n}$ and $\left(\tilde{u}_{i}^{(n)}\right)_{n}$ with $u_{i}^{(n)} \neq \tilde{u}_{i}^{(n)}, i=1,2$. This relation is still valid in the limit case; i.e., for $n \rightarrow \infty$ we have $u_{i}^{(\infty)} \neq \tilde{u}_{i}^{(\infty)}$ (unless $V_{1}$ and $V_{2}$ are disjoint), although $u_{i}^{(\infty)}$ and $\tilde{u}_{i}^{(\infty)}$ are minimizers of the same minimization problem; see Proposition 3.3. This behavior can be attributed to the fact that $J_{\alpha_{1}, \alpha_{2}}$ is not strictly convex and, thus, has in general more than one minimizer.

Next we provide an estimate for the distance between an accumulation point generated by either of the algorithms (3.1) or (3.2) and a minimizer of $J_{\alpha_{1}, \alpha_{2}}$.

Theorem 4.10. Assume that $u^{*}$ is a minimizer of $J_{\alpha_{1}, \alpha_{2}}$, and let $u^{(\infty)}$ be an accumulation point of the sequence $\left(u^{(n)}\right)_{n}$ generated by the algorithm in (3.1) or (3.2). Then we have that

1. $u^{(\infty)}$ is a minimizer of $J_{\alpha_{1}, \alpha_{2}}$, or

2. there exists a constant $\beta>0$ such that $\left\|u^{(\infty)}-u^{*}\right\|_{\ell^{2}(\Omega)} \leq \beta$, or

3. if $\alpha_{2}>0$ and $T_{2}^{*} T_{2}$ is positive definite in the direction $u^{(\infty)}-u^{*}$ with smallest eigenvalue $\sigma>0$, i.e., $\left\|T_{2}\left(u^{(\infty)}-u^{*}\right)\right\|_{\ell_{2}(\Omega)}^{2} \geq \sigma\left\|u^{(\infty)}-u^{*}\right\|_{\ell_{2}(\Omega)}^{2}$, then

$$
\left\|u^{(\infty)}-u^{*}\right\|_{\ell^{2}(\Omega)} \leq \frac{\|\hat{\eta}\|_{\ell^{2}(\Omega)}}{\alpha_{2} \sigma}
$$

where $\hat{\eta} \in \arg \min _{\eta \in \bigcup_{i=1}^{2}\left(\partial J_{\alpha_{1}, \alpha_{2}}\left(u^{(\infty)}\right) \cap V_{i}^{c}\right)}\|\eta\|_{\ell^{2}(\Omega)}$.

Proof. Let $\xi_{i}^{(n)} \in \arg \min _{\xi_{i} \in \mathcal{H}}\left\{J_{\alpha_{1}, \alpha_{2}}\left(\xi_{i}\right): \pi_{V_{i}^{c}} \xi_{i}=\pi_{V_{i}^{c}} b\right\}$ with $b \in V_{j}$ as in (3.3) for $i=1,2$ and $j \in\{1,2\} \backslash\{i\}$. Then, by [35, Thm. 2.1.4, p. 305] we have that $0 \in \partial J_{\alpha_{1}, \alpha_{2}}\left(\xi_{i}^{(n)}\right)+\eta_{i}^{(n)}$ for $\eta_{i} \in V_{i}^{c}$ and $i=1,2$. In other words, for example, for $i=1$ we have that $\xi_{1}^{(n)}-\tilde{u}_{2}^{(n-1)}=: u_{1}^{(n)} \in$ $\arg \min _{u_{1} \in V_{1}} J_{\alpha_{1}, \alpha_{2}}\left(u_{1}+\tilde{u}_{2}^{(n-1)}\right)$. Since $\left(\xi_{i}^{(n)}\right)_{n}$ is bounded, $\left(\partial J_{\alpha_{1}, \alpha_{2}}\left(\xi_{i}^{(n)}\right)\right)_{n}$ is bounded as well, and (cf. [44, Thm. 24.4, p. 233]) there exists $\eta_{i}^{(\infty)} \in V_{i}^{c}$ such that $0 \in \partial J_{\alpha_{1}, \alpha_{2}}\left(\xi_{i}^{(\infty)}\right)+\eta_{i}^{(\infty)}$, where $\xi_{i}^{(\infty)}$ is an accumulation point of $\left(\xi_{i}^{(n)}\right)_{n}$. Hence, $u_{i}^{(\infty)}$ is optimal in $V_{i}$ for $i=1,2$. From Proposition 4.8 we get that if $u_{i}^{(\infty)}$ is optimal, then also $\tilde{u}_{i}^{(\infty)}$ is optimal. Hence for $i=1$ we have $\tilde{u}^{(\infty)}-\tilde{u}_{2}^{(\infty)}:=\tilde{u}_{1}^{(\infty)} \in \arg \min _{u_{1} \in V_{1}} J_{\alpha_{1}, \alpha_{2}}\left(u_{1}+\tilde{u}_{2}^{(\infty)}\right)$, which means that there exists $\hat{\eta}_{1} \in V_{1}^{c}$ such that $0 \in \partial J_{\alpha_{1}, \alpha_{2}}\left(\tilde{u}^{(\infty)}\right)+\hat{\eta}_{1}$. Similarly we get for $i=2$ that there exists $\hat{\eta}_{2} \in V_{2}^{c}$ such that $0 \in \partial J_{\alpha_{1}, \alpha_{2}}\left(u^{(\infty)}\right)+\hat{\eta}_{2}$. Note that for the sequential algorithm in (3.1) we have $\tilde{u}^{(\infty)} \neq u^{(\infty)}$ in general, while for the parallel algorithm in $(3.2)$ we have $\tilde{u}^{(\infty)}=u^{(\infty)}$. In the rest of the proof we denote by $u^{(\infty)}$ an accumulation point which fulfills at least one of the aforementioned inclusions.

By the definition of the subdifferential we obtain

$$
J_{\alpha_{1}, \alpha_{2}}\left(u^{(\infty)}\right) \leq J_{\alpha_{1}, \alpha_{2}}(v)+\left\langle\hat{\eta}, u^{(\infty)}-v\right\rangle_{\mathcal{H}} \leq J_{\alpha_{1}, \alpha_{2}}(v)+\|\hat{\eta}\|_{\ell^{2}(\Omega)}\left\|v-u^{(\infty)}\right\|_{\ell^{2}(\Omega)}
$$

for all $v \in \mathcal{H}$, where $\hat{\eta}=\arg \min _{\left.\eta \in \bigcup_{i=1}^{2}\left(\partial J_{\alpha_{1}, \alpha_{2}(u}(\infty)\right) \cap V_{i}^{c}\right)}\|\eta\|_{\ell^{2}(\Omega)}$.

Let $u^{*} \in \arg \min _{u \in \mathcal{H}} J_{\alpha_{1}, \alpha_{2}}(u)$. Then the optimality of $u^{*}$ yields that the directional derivative of $J_{\alpha_{1}, \alpha_{2}}$ at $u^{*}$ in any direction $s \in \mathcal{H}$ is nonnegative; i.e., $J_{\alpha_{1}, \alpha_{2}}^{\prime}\left(u^{*} ; s\right) \geq 0$. Set 
$P(\xi):=\alpha_{1}\left\|T_{1} \xi-g_{1}\right\|_{\ell^{1}(\Omega)}+\varphi(|\nabla \xi|)(\Omega)$. Then, by using Taylor's expansion, for $s \in \mathcal{H}$ we have that

$$
\begin{aligned}
J_{\alpha_{1}, \alpha_{2}}\left(u^{*}+s\right) & =\alpha_{2}\left\|T_{2} u^{*}-g_{2}\right\|_{\ell^{2}(\Omega)}^{2}+\left\langle s, 2 \alpha_{2} T_{2}^{*}\left(T_{2} u^{*}-g_{2}\right)\right\rangle_{\mathcal{H}}+\alpha_{2}\left\|T_{2} s\right\|_{\ell^{2}(\Omega)}^{2}+P\left(u^{*}+s\right) \\
& =J_{\alpha_{1}, \alpha_{2}}\left(u^{*}\right)+\left\langle s, 2 \alpha_{2} T_{2}^{*}\left(T_{2} u^{*}-g_{2}\right)\right\rangle_{\mathcal{H}}+P\left(u^{*}+s\right)-P\left(u^{*}\right)+\alpha_{2}\left\|T_{2} s\right\|_{\ell^{2}(\Omega)}^{2} .
\end{aligned}
$$

By using $P\left(u^{*}+s\right)-P\left(u^{*}\right) \geq P^{\prime}\left(u^{*} ; s\right)$, which easily follows from the convexity of $P$, we obtain that

$$
J_{\alpha_{1}, \alpha_{2}}\left(u^{*}+s\right) \geq J_{\alpha_{1}, \alpha_{2}}\left(u^{*}\right)+\left\langle s, 2 \alpha_{2} T_{2}^{*}\left(T_{2} u^{*}-g_{2}\right)\right\rangle_{\mathcal{H}}+P^{\prime}\left(u^{*} ; s\right)+\alpha_{2}\left\|T_{2} s\right\|_{\ell^{2}(\Omega)}^{2} .
$$

Since $J_{\alpha_{1}, \alpha_{2}}^{\prime}\left(u^{*} ; s\right)=\left\langle s, 2 \alpha_{2} T_{2}^{*}\left(T_{2} u^{*}-g_{2}\right)\right\rangle_{\mathcal{H}}+P^{\prime}\left(u^{*} ; s\right) \geq 0$ and $\alpha_{2}\left\|T_{2} s\right\|_{\ell^{2}(\Omega)}^{2} \geq 0$ there exists a constant $\rho \geq 0$ such that $J_{\alpha_{1}, \alpha_{2}}\left(u^{*}+s\right)=J_{\alpha_{1}, \alpha_{2}}\left(u^{*}\right)+\rho$.

1. If $\rho=0$ for $s:=u^{(\infty)}-u^{*}$, then it immediately follows that $u^{*}+s=u^{(\infty)}$ is a minimizer of $J_{\alpha_{1}, \alpha_{2}}$.

2. If $\rho>0$ for $s:=u^{(\infty)}-u^{*}$, then from the coercivity condition we obtain that there exists a constant $\beta>0$ such that $0<\left\|u^{(\infty)}-u^{*}\right\|_{\ell_{2}(\Omega)} \leq \beta<+\infty$.

3. If, additionally, $\alpha_{2}>0$ and there exists a constant $\sigma>0$ such that $\| T_{2}\left(u^{(\infty)}-\right.$ $\left.u^{*}\right)\left\|_{\ell_{2}(\Omega)}^{2} \geq \sigma\right\| u^{(\infty)}-u^{*} \|_{\ell_{2}(\Omega)}^{2}$, then we get from (4.22) that

$$
J_{\alpha_{1}, \alpha_{2}}\left(u^{*}+s\right) \geq J_{\alpha_{1}, \alpha_{2}}\left(u^{*}\right)+\alpha_{2} \sigma\left\|u^{(\infty)}-u^{*}\right\|_{\ell_{2}(\Omega)}^{2} .
$$

Setting $v=u^{*}$ in (4.21) and using the inequality in (4.23) yields

$$
J_{\alpha_{1}, \alpha_{2}}\left(u^{*}\right)+\alpha_{2} \sigma\left\|u^{(\infty)}-u^{*}\right\|_{\ell^{2}(\Omega)}^{2} \leq J_{\alpha_{1}, \alpha_{2}}\left(u^{(\infty)}\right) \leq J_{\alpha_{1}, \alpha_{2}}\left(u^{*}\right)+\|\hat{\eta}\|_{\ell^{2}(\Omega)}\left\|u^{*}-u^{(\infty)}\right\|_{\ell^{2}(\Omega)}
$$

and consequently

$$
\left\|u^{(\infty)}-u^{*}\right\|_{\ell^{2}(\Omega)} \leq \frac{\|\hat{\eta}\|_{\ell^{2}(\Omega)}}{\alpha_{2} \sigma}
$$

We have the following immediate consequence of Theorem 4.10.

Corollary 4.11. Let the assumptions of Theorem 4.10 hold true. If $\left\|\eta_{i}^{\left(n_{\ell}\right)}\right\| \rightarrow 0$ for $\ell \rightarrow \infty$ along a suitable subsequence $\left(n_{\ell}\right)_{\ell}$ for at least one $i \in\{1,2\}$, then any accumulation point of the sequence $\left(u^{(n)}\right)_{n}$ generated by the algorithm in (3.1) or (3.2) is a minimizer of $J_{\alpha_{1}, \alpha_{2}}$.

4.2.2. A modified sequential subspace correction method. Note that the algorithm in (3.1) is a special case of a coordinate descent method, where the spaces $V_{i}$ are chosen in a cyclic manner; see $[48,49]$ for more details on different rules for choosing the subspaces. In the sense of coordinate descent methods, our algorithm in (3.1) can be written as follows.

CD-Algorithm: Choose $u^{(0)} \in \mathcal{H}$ and iterate for $n=0,1,2, \ldots$

(1) choose a nonempty space $V_{n} \subset \mathcal{H}$;

(2) compute $s^{(n)}=s_{T_{2}^{*} T_{2}}\left(u^{(n)}, V_{n}\right) \in \arg \min _{s}\left\{J_{\alpha_{1}, \alpha_{2}}\left(u^{(n)}+s\right)\right.$ s.t. $\left.\pi_{V_{n}^{c}} s=0\right\}$;

(3) set $u^{(n+1)}=u^{(n)}+s^{(n)}$. 
In step 2, the subscript $T_{2}^{*} T_{2}$ refers to the Hessian of the smooth part of $J_{\alpha_{1}, \alpha_{2}}$. Compared to [49], here we choose the step-size in the update of $u^{(n+1)}$ to be 1 , which is justified by (4.3) for $\alpha_{2}>0$. As already mentioned, there exist several different ways of choosing $V_{n}$ in each iteration. We suggest selecting $V_{n}$ such that

$$
\left\|s_{D}\left(u^{(n)}, V_{n}\right)\right\|_{\ell^{2}(\Omega)} \geq \nu\left\|s_{D}\left(u^{(n)}, \mathcal{H}\right)\right\|_{\ell^{2}(\Omega)},
$$

where $0<\nu \leq 1$ and $D: \mathcal{H} \rightarrow \mathcal{H}$ is positive definite and diagonal, i.e., there exists a $\tilde{D} \in \mathcal{H}$ associated with $D$ such that $D u=\tilde{D} \circ u$ (Hadamard product) with $[\tilde{D} \circ u](x)=\tilde{D}(x) u(x)$ for any $u \in \mathcal{H}$ and $x \in \Omega$. Here, the subscript $D$ indicates that $T_{2}^{*} T_{2}$, the Hessian of the smooth part of $J_{\alpha_{1}, \alpha_{2}}$, is replaced by $D$. This rule is called the Gauss-Southwell-r rule, which also allows the choice $V_{n}=\mathcal{H}$. With this selection rule for the subspaces, we are able to establish global convergence. The proof follows from Theorem 1 of [49].

Theorem 4.12. Assume $2 \alpha_{2}\left\|T_{2}\right\|^{2} \geq \underline{\lambda}>0$. Let $\left(u^{(n)}\right)_{n},\left(s^{(n)}\right)_{n}$ be sequences generated by the CD-Algorithm. If $\left(V_{n}\right)$ is chosen by the Gauss-Southwell-r rule with $D$ positive definite, diagonal, and bounded, i.e., there exists a $\bar{\delta}>0$ such that $\|D u\| \leq \bar{\delta}\|u\|$ for all $u \in \mathcal{H}$, then every cluster point of $\left(u^{(n)}\right)_{n}$ is a minimizer of $J_{\alpha_{1}, \alpha_{2}}$.

5. Application: Domain decomposition. The results of the previous sections are valid for any splitting of the function space $\mathcal{H}$. We concentrate now on decompositions which split the spatial domain into two subdomains. But let us emphasize that a generalization to a splitting into more domains is straightforward.

5.1. Overlapping domain decomposition. In this section we focus on an overlapping domain decomposition method. Thus we want to minimize (4.1) by decomposing $\Omega$ into two overlapping subdomains $\Omega_{1}$ and $\Omega_{2}$ such that $\Omega=\Omega_{1} \cup \Omega_{2}$ and $\Omega_{1} \cap \Omega_{2} \neq \emptyset$. By $\Gamma_{1}$ we denote the interface between $\Omega_{1}$ and $\Omega_{2} \backslash \Omega_{1}$ and by $\Gamma_{2}$ the interface between $\Omega_{2}$ and $\Omega_{1} \backslash \Omega_{2}$. For consistency with the definitions of the gradient and divergence operators, we assume that the subdomains $\Omega_{i}$ as well as $\Omega$ are discrete $d$-orthotopes. We stress that this is by no means a restriction, but it simplifies the presentation. Associated wih the splitting of $\Omega$ we define $V_{i}=\left\{u \in \mathcal{H}: \operatorname{supp}(u) \subset \Omega_{i}\right\}$. One aims now to minimize $J_{\alpha_{1}, \alpha_{2}}$ by the alternating algorithm in (3.1) or the parallel algorithm in (3.2). Note that the respective subspace minimization problems are constrained optimization problems of the type (3.3). In particular, for the alternating algorithm we have in $V_{1}$

$$
\min _{v \in \mathcal{H}} J_{\alpha_{1}, \alpha_{2}}(v) \text { s.t. } \pi_{V_{1}^{c}} v=\pi_{V_{1}^{c}} \tilde{u}_{2}^{(n)}
$$

and in $V_{2}$ we have

$$
\min _{v \in \mathcal{H}} J_{\alpha_{1}, \alpha_{2}}(v) \text { s.t. } \pi_{V_{2}^{c}} v=\pi_{V_{2}^{c}} u_{1}^{(n+1)},
$$

while for the parallel algorithm the minimization in $V_{1}$ is again (5.1), and in $V_{2}$ it changes to

$$
\min _{v \in \mathcal{H}} J_{\alpha_{1}, \alpha_{2}}(v) \text { s.t. } \pi_{V_{2}^{c} v}=\pi_{V_{2}^{c}} \tilde{u}_{1}^{(n)}
$$


5.2. Nonoverlapping domain decomposition. In the nonoverlapping domain decomposition method we want to minimize (4.1) by decomposing $\Omega$ into two nonoverlapping subdomains $\Omega_{1}$ and $\Omega_{2}$ such that $\Omega=\Omega_{1} \cup \Omega_{2}$ and $\Omega_{1}=\Omega \backslash \Omega_{2}$. For consistency with the definitions of the gradient and divergence operators, we again assume that the subdomains $\Omega_{i}$ as well as $\Omega$ are discrete $d$-orthotopes. Associated with the splitting of $\Omega$ we define by $V_{i}=\left\{u \in \mathcal{H}: \operatorname{supp}(u) \subset \Omega_{i}\right\}$ the function space of the subdomain $\Omega_{i}$. Then we minimize $J_{\alpha_{1}, \alpha_{2}}$ either by the parallel algorithm in (3.2) or by the alternating algorithm in (3.1), which specifies to the following.

Choose an initial $u^{(0)}=: u_{1}^{(0)}+u_{2}^{(0)} \in V_{1} \oplus V_{2}$, for example $u^{(0)}=0$, and iterate

$$
\left\{\begin{aligned}
u_{1}^{(n+1)} \leftarrow \arg \min _{u_{1} \in V_{1}} J_{\alpha_{1}, \alpha_{2}}\left(u_{1}+u_{2}^{(n)}\right), \\
u_{2}^{(n+1)} \leftarrow \arg \min _{u_{2} \in V_{2}} J_{\alpha_{1}, \alpha_{2}}\left(u_{1}^{(n+1)}+u_{2}\right), \\
u^{(n+1)} \leftarrow u_{1}^{(n+1)}+u_{2}^{(n+1)} .
\end{aligned}\right.
$$

The subspace optimization problems for the alternating version are

$$
\begin{gathered}
\min _{u_{1} \in V_{1}} J_{\alpha_{1}, \alpha_{2}}\left(u_{1}+u_{2}^{(n)}\right)=\min _{u_{1} \in V_{1}} \alpha_{1}\left\|T_{1} u_{1}-\left(g_{1}-T_{1} u_{2}^{(n)}\right)\right\|_{\ell^{1}(\Omega)}+\alpha_{2}\left\|T_{2} u_{1}-\left(g_{2}-T_{2} u_{2}^{(n)}\right)\right\|_{\ell^{2}(\Omega)}^{2} \\
+\varphi\left(\left|\nabla\left(u_{1}+u_{2}^{(n)}\right)\right|\right)(\Omega)
\end{gathered}
$$

in $V_{1}$ and

$$
\begin{aligned}
\min _{u_{2} \in V_{2}} J_{\alpha_{1}, \alpha_{2}}\left(u_{1}^{(n+1)}+u_{2}\right)=\min _{u_{2} \in V_{2}} \alpha_{1} \| & T_{1} u_{2}-\left(g_{1}-T_{1} u_{1}^{(n+1)}\right) \|_{\ell^{1}(\Omega)} \\
& +\alpha_{2}\left\|T_{2} u_{2}-\left(g_{2}-T_{2} u_{1}^{(n+1)}\right)\right\|_{\ell^{2}(\Omega)}^{2}+\varphi\left(\left|\nabla\left(u_{1}^{(n+1)}+u_{2}\right)\right|\right)(\Omega)
\end{aligned}
$$

in $V_{2}$. Upon adjusting notation, for the parallel algorithm the subspace minimization problems look similar.

A very special situation occurs when $\left(T_{1} u_{2}\right)(x)=0$ for all $x \in \Omega$ and $\left(T_{2} u_{1}\right)(x)=0$ for all $x \in \Omega$, which is the case when $T_{i}=1_{\Omega_{i}} \tilde{T}_{i}$ with $\tilde{T}_{i}: \mathcal{H} \rightarrow \mathcal{H}$ such that for all $v_{j} \in V_{j}$ we have $\tilde{T}_{i} v_{j} \in V_{j}$ for $j=1,2$ and $i=1,2$ (e.g., $\tilde{T}_{i}=I$ or $\tilde{T}_{i}=1_{\Omega \backslash K}$ with $K \subset \Omega$ ). Then the above subspace minimization problems simplify to

$$
\min _{u_{1} \in V_{1}} J_{\alpha_{1}, \alpha_{2}}\left(u_{1}+u_{2}^{(n)}\right)=\min _{u_{1} \in V_{1}} \alpha_{1}\left\|T_{1} u_{1}-g_{1}\right\|_{\ell^{1}(\Omega)}+\varphi\left(\left|\nabla\left(u_{1}+u_{2}^{(n)}\right)\right|\right)(\Omega)
$$

in $V_{1}$ and

$$
\min _{u_{2} \in V_{2}} J_{\alpha_{1}, \alpha_{2}}\left(u_{1}^{(n+1)}+u_{2}\right)=\min _{u_{2} \in V_{2}} \alpha_{2}\left\|T_{2} u_{2}-g_{2}\right\|_{\ell^{2}(\Omega)}^{2}+\varphi\left(\left|\nabla\left(u_{1}^{(n+1)}+u_{2}\right)\right|\right)(\Omega)
$$

in $V_{2}$.

5.3. Numerical implementation. In this section we propose an implementation of the domain decomposition algorithms in (3.1) and (3.2) when the domain is split into overlapping and nonoverlapping subdomains for the particular case $\varphi(|\nabla u|)(\Omega)=|\nabla u|(\Omega)$ (total variation of $u$ in $\Omega$ ), i.e., for the minimization problem

$$
\arg \min _{u \in \mathcal{H}} \alpha_{1}\left\|T_{1} u-g_{1}\right\|_{\ell^{1}(\Omega)}+\alpha_{2}\left\|T_{2} u-g_{2}\right\|_{\ell^{2}(\Omega)}^{2}+|\nabla u|(\Omega) .
$$


As our implementation works for both the nonoverlapping and overlapping domain decomposition algorithm, we use the notation for the overlapping splitting only. More precisely, in the nonoverlapping case $\Omega_{i} \backslash \Omega_{\check{i}}$ turns out to be all of $\Omega_{i}$ for $i=1,2$ and $\check{i} \in\{1,2\} \backslash\{i\}$, and for a nonoverlapping decomposition we have that $\Gamma_{1}=\Gamma_{2}$ is the interface between $\Omega_{1}$ and $\Omega_{2}$.

5.3.1. Implementation of the domain decomposition algorithms. If we compute the minimizer of the functional (4.1) either via the sequential or parallel nonoverlapping domain decomposition algorithm or via the sequential or parallel overlapping domain decomposition algorithm, then, on each subdomain, we have to solve a problem of the type

$$
\min _{\xi \in \mathcal{H}} J_{\alpha_{1}, \alpha_{2}}(\xi) \text { s.t. } A \xi=b,
$$

where $A: \mathcal{H} \rightarrow \mathcal{H}$ is a linear operator or more precisely an orthogonal projection, i.e., $A=\pi_{V_{i}^{c}}$ for $i=1,2$. There exist several numerical methods which efficiently solve (5.6). Instances are the augmented Lagrangian method $[5,36]$ or its variations known as Bregman iterations $[43,55,56]$ because of their relation to the Bregman distance [8].

Note that the functional $J_{\alpha_{1}, \alpha_{2}}$ is defined on all of $\Omega$. We describe now how one may reduce the dimensionality of the subproblems and solve the resulting problems.

Subspace minimization. We consider the minimization problem in $\Omega_{1}$ written as

$\min _{u_{1} \in V_{1}} J_{\alpha_{1}, \alpha_{2}}\left(u_{1}+u_{2}\right)=\alpha_{1}\left\|T_{1}\left(u_{1}+u_{2}\right)-g_{1}\right\|_{\ell^{1}(\Omega)}+\alpha_{2}\left\|T_{2}\left(u_{1}+u_{2}\right)-g_{2}\right\|_{\ell^{2}(\Omega)}^{2}+\left|\nabla\left(u_{1}+u_{2}\right)\right|(\Omega)$,

where $u_{2} \in V_{2}$ is fixed. In order to compute a minimizer of (5.7) we use the discrete analogue of the algorithm described in section 2.2 in $V_{1}$ : (i) we introduce a new variable $v=T_{1}\left(u_{1}+u_{2}\right)-g_{1}$, (ii) we regularize the functional in (5.7) as in (2.2), (iii) analogously to (2.3), we solve

$$
\min _{v \in \mathcal{H}} \alpha_{1}\|v\|_{\ell^{1}(\Omega)}+\frac{1}{2 \gamma}\left\|T_{1} u-g_{1}-v\right\|_{\ell^{2}(\Omega)}^{2},
$$

and (iv) instead of (2.5) we minimize

$$
\min _{u_{1} \in V_{1}} \frac{1}{2 \gamma}\left\|T_{1} u_{1}-\left(g_{1}-T_{1} u_{2}\right)-v\right\|_{\ell^{2}(\Omega)}^{2}+\alpha_{2}\left\|T_{2} u_{1}-\left(g_{2}-T_{2} u_{2}\right)\right\|_{\ell^{2}(\Omega)}^{2}+\left|\nabla\left(u_{1}+u_{2}\right)\right|(\Omega) .
$$

Similarly to (2.7), an approximate solution to (5.9) may be computed by the following iterative algorithm: Initialize $u_{1}^{(0)} \in V_{1}$ and iterate

$$
u_{1}^{(\ell+1)} \in \arg \min _{u_{1} \in V_{1}} S\left(u_{1}+u_{2}, u_{1}^{(\ell)}+u_{2}\right) \quad \text { for } \ell \geq 0 .
$$

Thanks to the splitting property of the total variation, i.e.,

$$
\left|\nabla\left(u_{1}+u_{2}\right)\right|(\Omega)=\left|\nabla\left(u_{1}+u_{2}\right)\right|\left(\Omega_{1} \cup \tilde{\Omega}_{2}\right)+f\left(u_{2}\right),
$$

where $f$ is a function independent of $u_{1}$ (see [2]), we can restrict (5.10) to $\Omega_{1} \cup \tilde{\Omega}_{2}$, where $\tilde{\Omega}_{2} \subset \Omega_{2} \backslash \Omega_{1}$ is a small neighborhood strip around the interface $\Gamma_{1}$. Hence the minimization problem in (5.10) is equivalent to

$$
\min _{u_{1} \in V_{1}}\left\|u_{1}+u_{2}-\frac{\gamma}{1+2 \alpha_{2} \gamma}\left(\frac{1}{\gamma} z_{1}+2 \alpha_{2} z_{2}\right)\right\|_{\ell^{2}\left(\Omega_{1} \cup \tilde{\Omega}_{2}\right)}^{2}+\frac{2 \gamma}{1+2 \alpha_{2} \gamma}\left|\nabla\left(u_{1}+u_{2}\right)\right|\left(\Omega_{1} \cup \tilde{\Omega}_{2}\right)
$$


with $z_{1}=u_{1}^{(\ell)}+u_{2}+T_{1}^{*}\left(g_{1}+v-T_{1} u_{1}^{(\ell)}-T_{1} u_{2}\right)$ and $z_{2}=u_{1}^{(\ell)}+u_{2}+T_{2}^{*}\left(g_{2}-T_{2} u_{1}^{(\ell)}-T_{2} u_{2}\right)$. We compute a solution of this problem by solving the constrained minimization problem

$$
\begin{aligned}
& \min _{\xi_{1} \in V_{1} \oplus \tilde{V}_{2}}\left\|\xi_{1}-\frac{\gamma}{1+2 \alpha_{2} \gamma}\left(\frac{1}{\gamma} z_{1}+2 \alpha_{2} z_{2}\right)\right\|_{\ell^{2}\left(\Omega_{1} \cup \tilde{\Omega}_{2}\right)}^{2}+\frac{2 \gamma}{1+2 \alpha_{2} \gamma}\left|\nabla \xi_{1}\right|\left(\Omega_{1} \cup \tilde{\Omega}_{2}\right) \\
& \text { s.t. } \pi_{\tilde{V}_{2}} \xi_{1}=\pi_{\tilde{V}_{2}} u_{2},
\end{aligned}
$$

where $\tilde{V}_{2}:=\left\{v \in \mathcal{H}: \operatorname{supp}(v) \subset \tilde{\Omega}_{2}\right\}$. Note that $\xi_{1}$ is optimal if and only if $u_{1}=\xi_{1}-\pi_{\tilde{V}_{2}} u_{2} \in V_{1}$ is optimal. We compute a minimizer of the problem in (5.12), which is of the form (5.6), by the Bregmanized operator splitting-split Bregman algorithm [38].

Remark 5.1. The minimization problem (2.3) and (5.8) can be solved very efficiently on the whole domain $\Omega$, since we only have to perform a soft-thresholding. On the other hand, we could restrict the constrained $L^{2}$-TV minimization (5.12) to the domain $\Omega_{1} \cup \tilde{\Omega}_{2}$, i.e., on $\Omega_{1}$ plus a small stripe around the interface. This is possible since we freed $u_{1}$ from the operators $T_{1}$ and $T_{2}$ and because of the splitting property of the total variation (5.11).

Remark 5.2 ( $L^{1}$-TV minimization). In the case when $\alpha_{2}=0$ and $\alpha_{1}>0$, i.e., the minimization problem in (5.5) becomes the $L^{1}$-TV model, each subspace minimization problem can be computed in the same way as described above. In fact, we first minimize (5.8) and then solve the constrained minimization problem (5.12), which simplifies to

$$
\min _{\xi_{1} \in V_{1} \oplus \tilde{V}_{2}}\left\|\xi_{1}-z_{1}\right\|_{\ell^{2}\left(\Omega_{1} \cup \tilde{\Omega}_{2}\right)}^{2}+2 \gamma\left|\nabla \xi_{1}\right|\left(\Omega_{1} \cup \tilde{\Omega}_{2}\right) \quad \text { s.t. } \pi_{\tilde{V}_{2}} \xi_{1}=\pi_{\tilde{V}_{2}} u_{2} .
$$

Remark 5.3 (denoising). If $T_{1}=T_{2}=I$, then we do not need surrogate functionals, and hence we do not have to perform the iterative algorithm (5.10). Instead we restrict (5.9) directly to $\Omega_{1} \cup \tilde{\Omega}_{2}$ and solve the following constrained minimization problem:

$$
\begin{aligned}
& \min _{\xi_{1} \in V_{1} \oplus \tilde{V}_{2}}\left\|\xi_{1}-\frac{\gamma}{1+2 \alpha_{2} \gamma}\left(\frac{1}{\gamma}\left(g_{1}+v\right)+2 \alpha_{2} g_{2}\right)\right\|_{\ell^{2}\left(\Omega_{1} \cup \tilde{\Omega}_{2}\right)}^{2}+\frac{2 \gamma}{1+2 \alpha_{2} \gamma}\left|\nabla \xi_{1}\right|\left(\Omega_{1} \cup \tilde{\Omega}_{2}\right) \\
& \text { s.t. } \pi_{\tilde{V}_{2}} \xi_{1}=\pi_{\tilde{V}_{2}} u_{2} .
\end{aligned}
$$

The minimization problem in $\Omega_{2}$ can be solved in the same way by adjusting the notation accordingly.

5.3.2. A special case. The implementation of the special case $T_{i}=1_{\Omega_{i}} \tilde{T}_{i}$ and $g_{i}=1_{\Omega_{i}} \tilde{g}_{i}$, where $\tilde{T}_{i}: \mathcal{H} \rightarrow \mathcal{H}$ and $\tilde{g}_{i} \in \mathcal{H}$, for $i=1,2$, is considered next. Note that the case considered here is more general than the situation discussed in section 5.2. The minimization problem in (5.5) can be written as

$$
\min _{u \in \mathcal{H}} \alpha_{1}\left\|\tilde{T}_{1} u-\tilde{g}_{1}\right\|_{\ell^{1}\left(\Omega_{1}\right)}+\alpha_{2}\left\|\tilde{T}_{2} u-\tilde{g}_{2}\right\|_{\ell^{2}\left(\Omega_{2}\right)}^{2}+|\nabla u|(\Omega) .
$$

When we solve this problem via one of the suggested domain decomposition methods, then on each subdomain we have to compute the minimizer of a constrained optimization problem. For example, in $\Omega_{1}$ we have

$$
\min _{u_{1} \in V_{1}} \alpha_{1}\left\|\tilde{T}_{1}\left(u_{1}+u_{2}\right)-\tilde{g}_{1}\right\|_{\ell^{1}\left(\Omega_{1}\right)}+\alpha_{2}\left\|\tilde{T}_{2}\left(u_{1}+u_{2}\right)-\tilde{g}_{2}\right\|_{\ell^{2}\left(\Omega_{2}\right)}^{2}+\left|\nabla\left(u_{1}+u_{2}\right)\right|(\Omega) .
$$


A solution of this problem can be obtained as described above in section 5.3.1. However, there exists a more efficient way of solving the problem in $\Omega_{1}$. This strategy, however, is not applicable for the minimization in $\Omega_{2}$ due to the special structure of the minimization problems with the $\ell^{1}$-norm defined only on $\Omega_{1}$. The main idea of this more efficient approach is the following:

1. Free $u_{1}$ from the influence of $\tilde{T}_{2}$ (respectively, $T_{2}$ ) by introducing a surrogate functional in a similar way as before; i.e., for $a \in V_{1}$ define

$$
\begin{aligned}
S\left(u_{1}+u_{2}, a\right):= & \alpha_{1}\left\|\tilde{T}_{1}\left(u_{1}+u_{2}\right)-\tilde{g}_{1}\right\|_{\ell^{1}\left(\Omega_{1}\right)}+\alpha_{2}\left\|T_{2}\left(u_{1}+u_{2}\right)-g_{2}\right\|_{\ell^{2}(\Omega)}^{2} \\
& +\left|\nabla\left(u_{1}+u_{2}\right)\right|(\Omega)+\alpha_{2}\left(\left\|u_{1}-a\right\|_{\ell^{2}(\Omega)}^{2}-\left\|T_{2}\left(u_{1}-a\right)\right\|_{\ell^{2}(\Omega)}^{2}\right) \\
= & \alpha_{1}\left\|\tilde{T}_{1}\left(u_{1}+u_{2}\right)-\tilde{g}_{1}\right\|_{\ell^{1}\left(\Omega_{1}\right)}+\alpha_{2}\left\|u_{1}-z_{2}\right\|_{\ell^{2}(\Omega)}^{2}+\left|\nabla\left(u_{1}+u_{2}\right)\right|(\Omega)+\psi,
\end{aligned}
$$

where $z_{2}=a+T_{2}^{*}\left(g_{2}-T_{2} u_{2}-T_{2} a\right)$ and $\psi$ is a function independent of $u_{1}$. Then compute an approximate solution of (5.14) by the following algorithm: Initialize $u_{1}^{(0)} \in V_{1}$ and iterate

$$
u_{1}^{(\ell+1)}=\arg \min _{u_{1} \in V_{1}} S\left(u_{1}+u_{2}, u_{1}^{(\ell)}\right) \quad \text { for } \ell \geq 0 .
$$

2. Thanks to (5.11), we can restrict the surrogate functional iteration to $\Omega_{1} \cup \tilde{\Omega}_{2}$.

3. In each surrogate iteration (5.15) one has to solve a constrained minimization problem. As an example, we describe here the Bregmanized operator splitting of [56]. For this purpose, let $\mu, \delta>0$, and initialize $\xi_{1}^{(0)} \in V_{1} \oplus \tilde{V}_{2}$ and $b^{(0)}=b=u_{2}$. Then for $k=0,1, \ldots$ solve

$$
\begin{aligned}
\xi_{1}^{(k+1)} & =\arg \min _{\xi_{1} \in V_{1} \oplus \tilde{V}_{2}} S\left(\xi_{1}, u_{1}^{(\ell)}\right)+\frac{\mu}{\delta}\left\|\xi_{1}-\left(\xi_{1}^{(k)}-\delta \pi_{\tilde{V}_{2}}^{*}\left(\pi_{\tilde{V}_{2}} \xi_{1}^{(k)}-b^{(k)}\right)\right)\right\|_{\ell^{2}\left(\Omega_{1} \cup \tilde{\Omega}_{2}\right)}^{2}, \\
b^{(k+1)} & =b^{(k)}-\pi_{\tilde{V}_{2}} \xi_{1}^{(k+1)}+b .
\end{aligned}
$$

4. Solve the minimization problem in (5.16) by the algorithm introduced in section 2.2.

Remark 5.4. Practically it seems that recomputing the Bregman update outside of the algorithm of section 2.2 is preferable (to computing the update inside the algorithm of section 2.2, as is done in section 5.3.1), as the resulting overall algorithm seems to converge faster according to our numerical practice.

Remark 5.5. In the case when $\tilde{T}_{1}=\tilde{T}_{2}=I$ and $\Omega_{1} \cap \Omega_{2}=\emptyset$, on each domain we have to solve the following constrained minimization problems:

$$
\min _{u_{1} \in V_{1} \oplus \tilde{V}_{2}} \alpha_{1}\left\|u_{1}-\tilde{g}_{1}\right\|_{\ell^{1}\left(\Omega_{1} \cup \tilde{\Omega}_{2}\right)}+\left|\nabla\left(u_{1}+u_{2}\right)\right|\left(\Omega_{1} \cup \tilde{\Omega}_{2}\right) \quad \text { s.t. } \pi_{\tilde{V}_{2}} u_{1}=0
$$

and

$$
\min _{u_{2} \in V_{2} \oplus \tilde{V}_{1}} \alpha_{2}\left\|u_{2}-\tilde{g}_{2}\right\|_{\ell^{2}\left(\Omega_{2} \cup \tilde{\Omega}_{1}\right)}^{2}+\left|\nabla\left(u_{1}+u_{2}\right)\right|\left(\Omega_{2} \cup \tilde{\Omega}_{1}\right) \quad \text { s.t. } \pi_{\tilde{V}_{1}} u_{2}=0,
$$


where $u_{1} \in V_{1}$ is fixed in (5.18) and $\tilde{\Omega}_{1}$ is defined analogously to $\tilde{\Omega}_{2}$. The subspace minimization problem (5.18) can be solved, for example, by the Bregmanized operator splitting-split Bregman algorithm [38], while one may solve (5.17) as suggested in this section above starting at point 3.

Due to the structure of the problem, for the minimization in $\Omega_{2}$ this approach is not applicable, and hence we suggest using the strategy of section 5.3.1.

5.4. Numerical experiments. In the following we present numerical experiments for the proposed sequential and parallel algorithms. In particular, we show applications in image denoising, inpainting, and deblurring. The values of the parameters $\alpha_{1}$ and $\alpha_{2}$ in the objective functional (5.5) are chosen according to the application and experimentally; i.e., we choose the values which give a good compromise between visual quality and computational time of the algorithm. We emphasize that the optimal selection of $\alpha_{1}$ and $\alpha_{2}$ is an interesting research topic in its own right but goes beyond the scope of the present paper. However, it has been shown in several examples - see $[9,41,42]$ - that if only salt-and-pepper noise is present in an image, then the $L^{1}$-TV model outperforms the $L^{2}$-TV model. Hence we use the pure $L^{1}$-TV model when only salt-and-pepper noise has corrupted the image of interest, whereas we use the pure $L^{2}$-TV model when only Gaussian noise is present.

5.4.1. Numerical results-Sequential algorithms. In our numerical experiments we terminate our sequential algorithms (3.1) and (5.2) as soon as the norm of the difference of two successive iterates drops below a certain threshold. More precisely, we use as a stopping criterion $\left\|u^{(n)}-u^{(n+1)}\right\|<10^{-6}$, which seems suitable for our purposes. In fact, if our algorithm converges at least linearly, i.e., if there exist an $\varepsilon \in(0,1)$ and an $m>0$ such that for all $n \geq m$ we have $\left\|u^{(n+1)}-u^{(\infty)}\right\| \leq \varepsilon\left\|u^{(n)}-u^{(\infty)}\right\|$, the above stopping criterion ensures that the distance between our obtained result $u$ and $u^{(\infty)}$ is $\left\|u-u^{(\infty)}\right\| \leq \frac{10^{-6} \varepsilon}{1-\varepsilon}$. Moreover, if we set $\alpha_{2}>0$, then we depict the minimal norm of Lagrange multipliers $\eta^{(n)}:=\min _{i}\left\{\left\|\eta_{i}^{(n)}\right\|_{\ell^{2}(\Omega)}\right\}$, which-according to Corollary 4.11 (see also Theorem 4.10)-indicates how close the computed solution is to the real global solution. In fact, when the minimal norm of Lagrange multipliers tends to zero numerically, then the associated domain decomposition algorithm indeed converges (along a subsequence) to the global solution; see Figures 4(c)-(d), 6(c)-(d), and $8(\mathrm{c})$.

We apply the overlapping and nonoverlapping domain decomposition algorithm in (3.1) to the image shown in Figure 4(a) by decomposing the image domain into two overlapping or nonoverlapping subdomains. This image of size $167 \times 270$ pixels has partly lost data (black heart) while it is also corrupted by $10 \%$ salt-and-pepper noise (i.e., $10 \%$ of the pixels are either flipped to black or white) and by Gaussian white noise with zero mean and variance 0.03. In this example the operators $T_{1}$ and $T_{2}$ act as $T_{i} u=1_{\Omega \backslash K} u$ for $i=1,2$, where $\Omega$ denotes the image domain and $K \subset \Omega$ the set in which the original image content got lost. The parameters $\alpha_{1}$ and $\alpha_{2}$ are chosen to be 0.4 , while $\gamma=0.01, \mu=1$, and $\delta=0.99$. In Figure 4(b) we depict the result computed by the overlapping domain decomposition algorithm. Since $\alpha_{2}$ is chosen to be positive, the progress of the minimal norm of the Lagrange multipliers allows us to check whether the iterates converge to the minimizer of the global functional. In fact, we see in Figure 4(c) and (d) that the minimal norm of the Lagrange multipliers converges to 0, and 


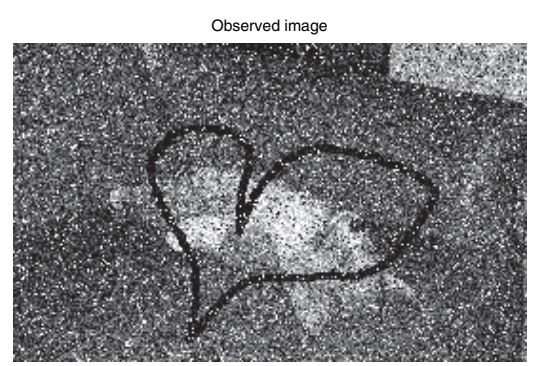

(a)

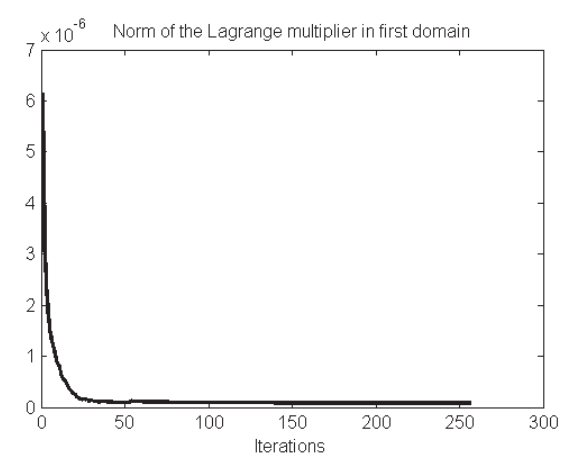

(c)

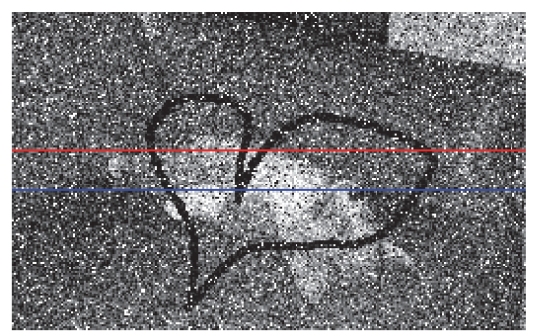

(e)

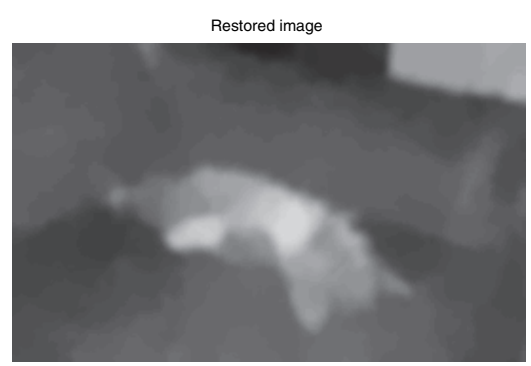

(b)

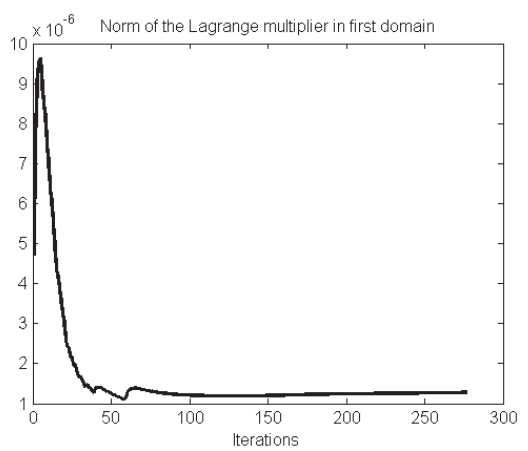

(d)

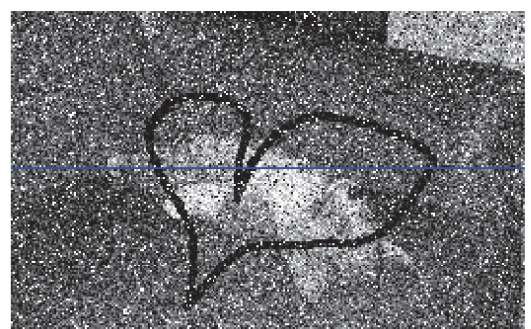

(f)

Figure 4. Domain decomposition for $L^{1}-L^{2}-T V$ minimization. Parameters: $\alpha_{1}=\alpha_{2}=0.4, \gamma=0.01$, $\mu=1, \delta=0.99$, and ROF-problem solved via the split Bregman method with tolerance $10^{-3}$. In (a) we show an image of size $167 \times 270$ pixels with a missing part (black heart) and corrupted by $10 \%$ salt-and-pepper noise and Gaussian noise with zero mean and variance 0.03. The restored image is shown in (b). In (c) we depict the progress of the minimal norm of Lagrange multipliers $\eta^{(n)}$ obtained by overlapping domain decomposition, as depicted in (e), where the red and blue lines mark the interfaces of the domain decomposition patches, while in (d) we plot the one obtained by the nonoverlapping domain decomposition; see (f) with the highlighted partition.

hence the accumulation points of the sequence of iterates converge to the global minimizer.

In the next example we present the successful application of a domain decomposition for the problem of pure $L^{1}$-TV minimization. Figure $5(\mathrm{a})$ shows the previously used image rescaled to size $334 \times 540$ pixels, which is now corrupted by a Gaussian blur with a kernel size 


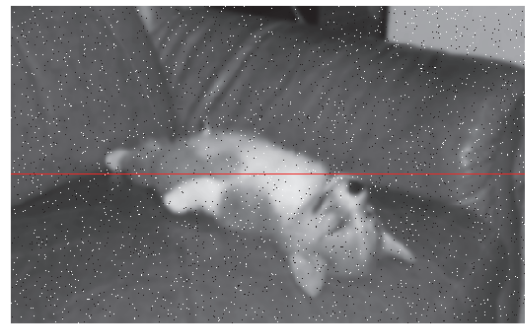

(a)

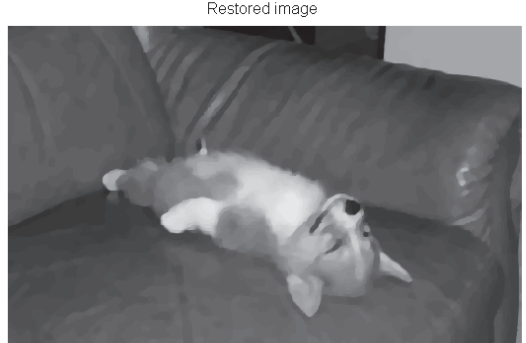

(b)

Figure 5. Nonoverlapping domain decomposition algorithm for $L^{1}-T V$ minimization. Parameters: $\alpha_{1}=$ $5 / 3, \gamma=0.01, \mu=1, \delta=0.99$, and ROF-problem solved via the split Bregman method with tolerance $10^{-4}$. In (a) we show an image of size $334 \times 540$ pixels which is corrupted by a Gaussian blur (size $15 \times 15$; standard deviation 2) and $2 \%$ salt-and-pepper noise. In this simulation the problem is split into two subproblems, where the decomposition is highlighted by the red line. The restored image is shown in (b).

of $15 \times 15$ pixels and standard deviation 2 and also by $2 \%$ salt-and-pepper noise. In order to restore the image we decompose the image domain into two nonoverlapping subdomains and solve the resulting problems on the respective subdomains alternatingly by the nonoverlapping domain decomposition algorithm (5.2). Since there is no Gaussian noise present, this is a typical example for $L^{1}$-TV minimization; i.e., we set $\alpha_{2}=0$ in (5.5). We choose $\alpha_{1}=\frac{5}{3}$, $\gamma=0.01, \mu=1$, and $\delta=0.99$ and obtain the image in Figure $5(\mathrm{~b})$.

Further we illustrate the successful application of the nonoverlapping domain decomposition algorithm (5.2) when both salt-and-pepper noise and Gaussian noise are present. In particular, we apply our algorithm to an image with a missing part which is corrupted by salt-and-pepper noise in the upper half while in the lower half only Gaussian noise is present. We are aware that this is a rather artificial example but very interesting from a numerical point of view. Note that since the total variation is nonlocal and hence nonadditive, it is not possible to obtain a correct global solution by just cutting the image into an upper and a lower part, computing the solutions separately, and then putting them together. However, since we are in the setting of the special situation of section 5.2, by using our nonoverlapping domain decomposition algorithm in (5.2), we are able to split the image into domains in which only one type of noise is present. Then we have to solve on each domain only either a constrained $L^{1}$-TV minimization problem (cf. (5.3)) or a constrained $L^{2}$-TV minimization problem (cf. (5.4)). These problems are in general easier to solve than the original $L^{1}-L^{2}$ TV problem. Figure $6(\mathrm{a})$ is such an image (size $167 \times 270$ pixels), which we restore by the nonoverlapping domain decomposition algorithm (5.2) with $\mu=100$ in the upper half and $\mu=1$ in the lower half, $\alpha_{1}=\frac{5}{3}, \alpha_{2}=\frac{50}{3}, \gamma=0.01$, and $\delta=0.99$. The computed result is shown in Figure 6(b). By depicting the minimal norm of Lagrange multipliers $\eta^{(n)}$, we check additionally whether the algorithm converges to the right solution. In Figure 6(c) we see the progress of the minimal norm of Lagrange multipliers for the image in Figure 6(a) with size $167 \times 270$ pixels. By improving the image resolution threefold, i.e., yielding an image with $501 \times 810$ pixels, we obtain a sequence $\left(\eta^{(n)}\right)_{n}$ which converges to a significantly smaller norm; 


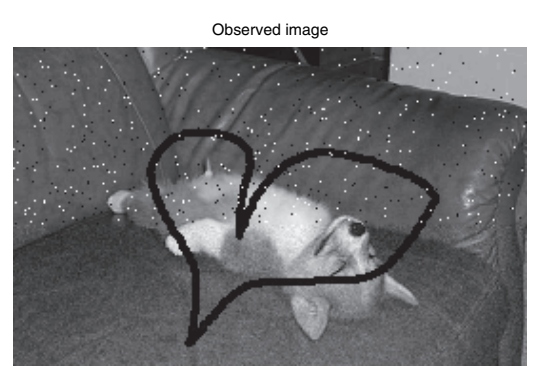

(a)

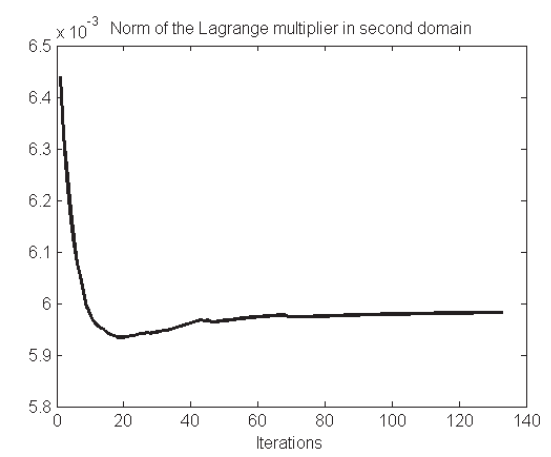

(c)

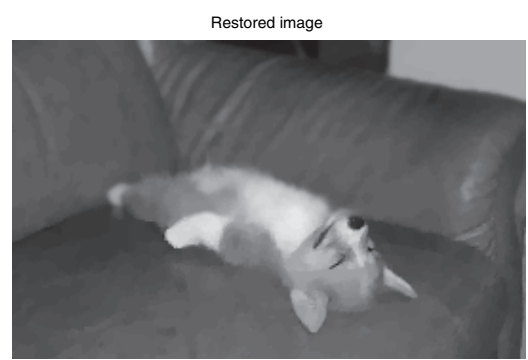

(b)

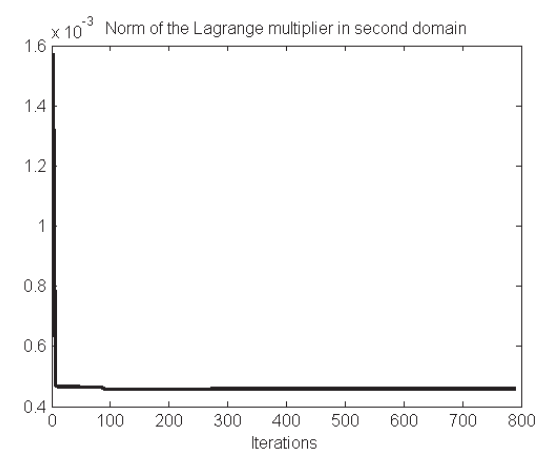

(d)

Figure 6. Nonoverlapping domain decomposition algorithm for $L^{1}-L^{2}-T V$ minimization. Parameters: $\alpha_{1}=\frac{5}{3}, \alpha_{2}=\frac{50}{3}, \gamma=0.01, \mu=100$ in $\Omega_{1}$ and $\mu=1$ in $\Omega_{2}, \delta=0.99$, and ROF-problem solved via the split Bregman method with tolerance $10^{-4}$. In (a) we show an image of size $167 \times 270$ pixels with a missing part (black heart) and corrupted by $2 \%$ salt-and-pepper noise and Gaussian noise with variance 0.001. The restored image is shown in (b). In (c) we depict the progress of the minimal norm of Lagrange multipliers $\eta^{(n)}$ as well as in (d) for a three times finer grid.

see Figure 6(d). If we keep increasing the image resolution, we observe that the sequence $\left(\eta^{(n)}\right)_{n}$ continues to converge to smaller and smaller values. This behavior may be attributed to the fact that the support of $\eta_{i}^{(n)}$ is confined to a small stripe of width two pixels, and this depends on the mesh size $h$.

With respect to the Gauss-Southwell- $r$ rule considered in section 4.2.2, we observe in our previous $L^{1}-L^{2}$-TV minimization examples that inequality $(4.24)$ is more likely to be satisfied with a fixed constant $\nu>0$ for overlapping rather than nonoverlapping domain decomposition. For instance, for the problem depicted in Figure 4, the Gauss-Southwell- $r$ rule is satisfied with $\nu \leq 0.03837$ along the iteration in the overlapping case. For the nonoverlapping decomposition one has $\nu \leq 0.000195$.

Sequential domain decomposition algorithm versus global $L^{1}-L^{2}-T V$ algorithm. We also compare the performance of the sequential domain decomposition algorithms with the $L^{1}-L^{2}-\mathrm{TV}$ algorithm, which solves the considered problems on all of $\Omega$ without any splitting into subdomains. Since we are comparing now the convergence speed of different algorithms, the 


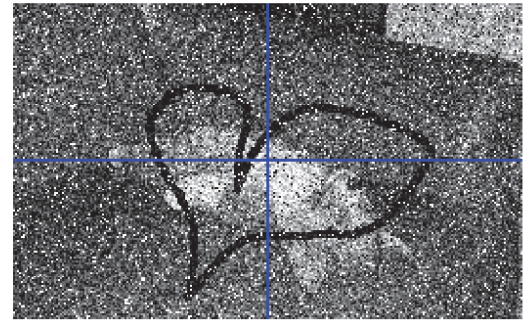

(a)

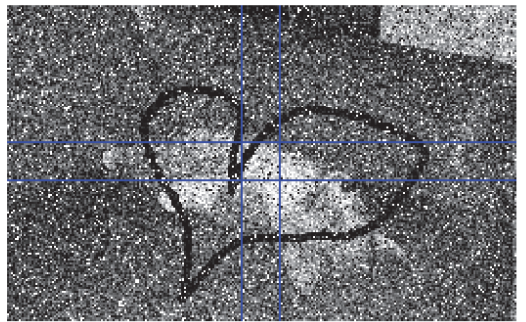

(b)

Figure 7. Splitting of the image in Figure 4 into four nonoverlapping and overlapping windows, as highlighted in blue.

Table 2

Restoration of the image in Figure 4: Computational performance (CPU time in seconds / the number of iterations / PSNR-value) for the global $L^{1}-L^{2}-T V$ algorithm and computational performance (CPU time in seconds / the number of iterations / PSNR-value / $\left.\min \left\{\left\|\eta_{1}\right\|^{2}, \ldots\left\|\eta_{D}\right\|^{2}\right\}\right)$ for the sequential domain decomposition algorithms with $\alpha_{1}=\alpha_{2}=0.4$ for different numbers of subdomains $(D=2,4,8)$ and overlapping sizes. The algorithms are stopped as soon as the energy reaches the significance level $J^{*}=0.098973$.

\begin{tabular}{|c|c|c|c|}
\hline \# domains & Nonoverlapping alg. & $\begin{array}{l}\text { Overlapping alg. } \\
\text { (overlap w } w=10 \\
\text { pixels) }\end{array}$ & $\begin{array}{l}\text { Overlapping alg. } \\
\text { (overlap } w=2 \text { pixels) }\end{array}$ \\
\hline$D=1\left(L^{1}-L^{2}-\mathrm{TV}\right.$ alg. $)$ & $471 \mathrm{~s} / 285$ it / 44.55 & & \\
\hline$D=2$ (stripe) & $\begin{array}{l}170 \mathrm{~s} / 41 \text { it / } 45.43 / \\
2.1 \cdot 10^{-7}\end{array}$ & $\begin{array}{l}162 \mathrm{~s} / 38 \text { it / } 45.13 / \\
1.1 \cdot 10^{-7}\end{array}$ & $\begin{array}{l}160 \mathrm{~s} / 40 \text { it / } 45.36 / \\
9.1 \cdot 10^{-8}\end{array}$ \\
\hline$D=4$ (stripe) & $\begin{array}{l}215 \mathrm{~s} / 41 \text { it / } 45.41 / \\
1.5 \cdot 10^{-7}\end{array}$ & $\begin{array}{l}225 \mathrm{~s} / 33 \text { it / } 45.18 / \\
1.3 \cdot 10^{-7}\end{array}$ & $\begin{array}{l}234 \mathrm{~s} / 39 \text { it / } 45.40 / \\
1.0 \cdot 10^{-7}\end{array}$ \\
\hline$D=4$ (window) & $\begin{array}{l}207 \mathrm{~s} / 41 \text { it / } 45.37 / \\
7.6 \cdot 10^{-8}\end{array}$ & $\begin{array}{l}168 \mathrm{~s} / 36 \text { it / } 45.02 / \\
8.0 \cdot 10^{-8}\end{array}$ & $\begin{array}{l}164 \mathrm{~s} / 40 \text { it / } 45.31 / \\
6.4 \cdot 10^{-8}\end{array}$ \\
\hline$D=8$ (stripe) & $\begin{array}{l}285 \mathrm{~s} / 41 \text { it } / 45.33 / \\
1.1 \cdot 10^{-7}\end{array}$ & $\begin{array}{l}213 \mathrm{~s} / 24 \text { it / } 45.01 / \\
7.8 \cdot 10^{-8}\end{array}$ & $\begin{array}{l}248 \mathrm{~s} / 35 \text { it / } 45.25 / \\
7.0 \cdot 10^{-8}\end{array}$ \\
\hline
\end{tabular}

stopping criterion used before is no longer suitable. Now we stop the algorithms as soon as the energy $J_{\alpha_{1}, \alpha_{2}}$ reaches a significance level $J^{*}$, i.e., when $J_{\alpha_{1}, \alpha_{2}}\left(u^{(n)}\right) \leq J^{*}$ for the first time. The level $J^{*}$ is chosen visually; i.e., we once restore the image of interest until we observe a visually satisfying restoration and record the associated energy value as $J^{*}$. Such a reasonable restoration can be obtained, for example, by running one of the algorithms until $\left\|u^{(n)}-u^{(n+1)}\right\|<10^{-6}$ for the first time, as was done above for the domain decomposition algorithms.

For our comparison we solved the problem associated with Figure 4 by considering splittings into $D=2,4,8$ overlapping and nonoverlapping stripes, as shown in Figure 4, and into $D=4$ overlapping and nonoverlapping windows, as depicted in Figure 7 . The width $w$ of the overlap is chosen to be 2 or 10 pixels. We stop the algorithms as soon as they reach the significant energy of $J^{*}=0.098973$. In Table 2 we summarize our findings. One clearly observes that the sequential domain decomposition algorithms are much faster than 
Table 3

Restoration of the image in Figure 5: Computational performance (CPU time in seconds / the number of iterations ( PSNR-value) for the global $L^{1}-T V$ algorithm and for the sequential domain decomposition algorithms with $\alpha_{1}=5 / 3$ for different numbers of subdomains $(D=2,4,8)$ and overlapping sizes. The algorithms are stopped as soon as the energy reaches the significance level $J^{*}=0.03052$.

\begin{tabular}{llll}
\hline \# domains & Nonoverlapping alg. & $\begin{array}{l}\text { Overlapping alg. } \\
\text { (overlap } w=10 \text { pixels) }\end{array}$ & $\begin{array}{l}\text { Overlapping alg. } \\
\text { (overlap } w=2 \text { pixels) }\end{array}$ \\
\hline$D=1$ ( $L^{1}$-TV alg.) & $153 \mathrm{~s} / 6$ it $/ 82.03$ & & \\
\hline$D=2$ (stripe) & $566 \mathrm{~s} / 10 \mathrm{it} / 81.18$ & $580 \mathrm{~s} / 9 \mathrm{it} / 81.15$ & $550 \mathrm{~s} / 9 \mathrm{it} / 81.22$ \\
\hline$D=4$ (stripe) & $445 \mathrm{~s} / 10 \mathrm{it} / 81.17$ & $484 \mathrm{~s} / 9 \mathrm{it} / 81.02$ & $430 \mathrm{~s} / 9$ it $/ 81.18$ \\
\hline$D=4$ (window) & $393 \mathrm{~s} / 5 \mathrm{it} / 80.26$ & $384 \mathrm{~s} / 5 \mathrm{it} / 80.31$ & $458 \mathrm{~s} / 5$ it $/ 80.29$ \\
\hline$D=8$ (stripe) & $508 \mathrm{~s} / 10 \mathrm{it} / 81.19$ & $467 \mathrm{~s} / 9 \mathrm{it} / 80.97$ & $486 \mathrm{~s} / 9$ it $/ 81.18$ \\
\hline
\end{tabular}

the global $L^{1}-L^{2}-\mathrm{TV}$ algorithm. Since the domain decomposition approach considered here is sequential, the convergence slows down in time when the number of subdomains is increased. Nevertheless, the number of iterations is nonincreasing with the number of subdomains, and even decreasing for the overlapping version. Moreover, in Table 2 we also present the minimal norm of Lagrange multipliers to indicate that in all of our experiments we are indeed very close to a global minimizer.

A different behavior can be observed for the deblurring problem of Figure 5, where only salt-and-pepper noise is present and therefore $\alpha_{2}$ is set to zero. We again tested the sequential domain decomposition algorithm for different splittings $(D=2,4,8)$ and compared the performance with the global algorithm; see Table 3. Now the sequential domain decomposition approach became computationally more expensive. However, we note that in general one should not expect that the sequential domain decomposition algorithms will always outperform the global one. Here this is particularly true, as $T_{1}$ is a nonlocal operator and the subproblems involve constraints, adding to the complexity of the solution approach.

In the next section we show the successful application of our solvers when both Gaussian noise as well as salt-and-pepper noise are present simultaneously (and in a nonseparated fashion) in an image; see the results depicted in Figure 8.

5.4.2. Numerical results-Parallel algorithms. Finally, we show the efficiency of the parallel algorithm in (3.2) for nonoverlapping and overlapping domain decomposition and compare its numerical performance with the $L^{1}-L^{2}-\mathrm{TV}$ algorithm introduced in section 2.2. Note that in the $L^{1}-L^{2}$-TV algorithm the problem is solved on all of $\Omega$ without any splitting into subdomains. In the domain decomposition algorithms we consider domain splittings into $D=4,8,16,32$ subdomains. Since we are comparing the convergence speed of different algorithms, we stop the algorithms as soon as the energy $J_{\alpha_{1}, \alpha_{2}}$ reaches a significance level $J^{*}$, as already described above.

For our comparison let us consider the image in Figure 8, which is of size $1920 \times 2576$ pixels and corrupted by Gaussian noise with standard deviation 0.01 as well as by $10 \%$ saltand-pepper noise on all $\Omega$; see Figure 8(a). In the domain decomposition algorithms as well as in the $L^{1}-L^{2}$-TV algorithm we denoise this image by choosing $\alpha_{1}=0.5$ and $\alpha_{2}=0.4$. The computations are done in MATLAB on a Linux cluster with 32 kernels, where each kernel has two processors and each processor four cores, i.e., on a computer with 256 cores, and the 

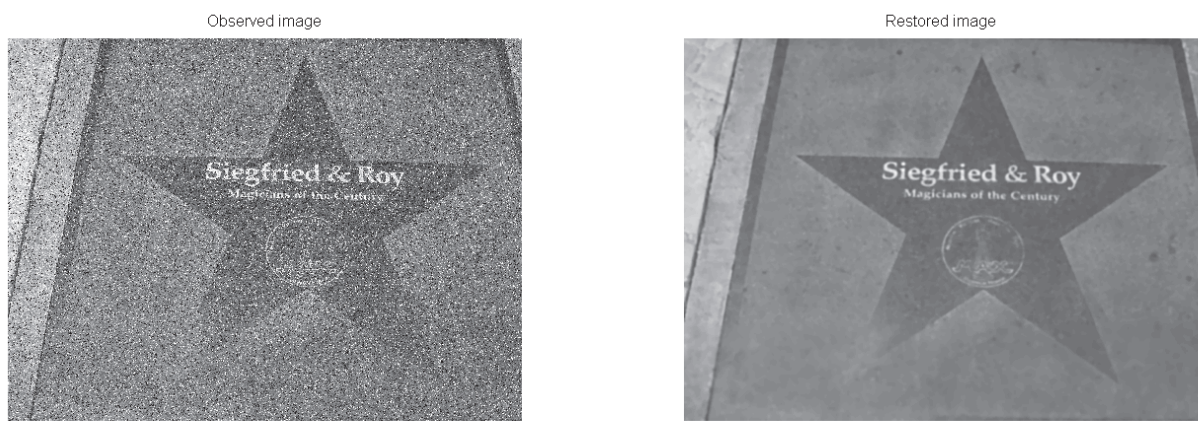

(a)

(b)

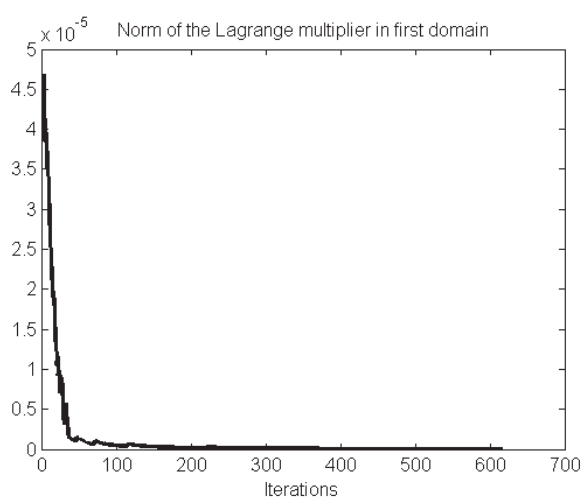

(c)

Figure 8. Parallel domain decomposition for $L^{1}-L^{2}-T V$ minimization of an image (size $1920 \times 2576$ pixels) corrupted by Gaussian noise with standard deviation 0.01 and $10 \%$ salt-and-pepper noise, (a). In (b) we show the image restored using the nonoverlapping domain decomposition algorithm for 8 domains with the following parameters: $\alpha_{1}=0.5, \alpha_{2}=0.4, \gamma=0.01, \mu=10, \delta=0.99$, and ROF-problem solved via the split Bregman method with tolerance $10^{-3}$. In (c) we depict the progress of the minimal norm of Lagrange multipliers $\eta^{(n)}$.

multithreading-option is activated such that all algorithms (including the $L^{1}-L^{2}$-TV algorithm without domain decomposition) take advantage of the parallel infrastructure offered by the hardware. For the domain decomposition algorithms we split the domain into nonoverlapping or overlapping strips. The overlap is chosen to be a stripe of width 10 pixels, i.e., the overlap is of size $10 \times 2576$ pixels. For different numbers of splittings we show in Table 4 the required computational time and the number of iterations until the algorithms reach the significant energy of $J^{*}=0.080041483485$ (see Figure 8(b) for the restored image). Note that the structure of the problems in the subdomains is different from that of the global problem. More precisely, on each subdomain we have to solve constrained minimization problems (cf. (3.3)), which are structurally more difficult to solve than just minimizing an energy as for the global problem. Hence by domain decomposition, on the one hand, we reduce the dimensionality of the problem, but, on the other hand, we increase the complexity on each subdomain. Additionally, we also have to take the communication time of the processors into account. 
Table 4

Denoising for the image in Figure 8: Computational performance (CPU time in seconds and the number of iterations) for the global $L^{1}-L^{2}-T V$ algorithm and for the parallel domain decomposition algorithms with $\alpha_{1}=0.5, \alpha_{2}=0.4$ for different numbers of subdomains $(D=4,8,16,32)$ and overlapping sizes.

\begin{tabular}{llll}
\hline \# domains & Nonoverlapping alg. & $\begin{array}{l}\text { Overlapping alg. } \\
\text { (overlap } 10 \times 2576 \\
\text { pixels) }\end{array}$ & $\begin{array}{l}\text { Overlapping alg. } \\
\text { (overlap 50 } 52576 \\
\text { pixels) }\end{array}$ \\
\hline$D=1\left(L^{1}-L^{2}-\mathrm{TV}\right.$ alg. $)$ & $7882 \mathrm{~s} / 698$ it & & \\
\hline$D=4$ & $5727 \mathrm{~s} / 617$ it & $5834 \mathrm{~s} / 607$ it & $5998 \mathrm{~s} / 561 \mathrm{it}$ \\
\hline$D=8$ & $5090 \mathrm{~s} / 618$ it & $5074 \mathrm{~s} / 596$ it & $5265 \mathrm{~s} / 499$ it \\
\hline$D=16$ & $5409 \mathrm{~s} / 588$ it & $5432 \mathrm{~s} / 560$ it & $5014 \mathrm{~s} / 371$ it \\
\hline$D=32$ & $6814 \mathrm{~s} / 586$ it & $6605 \mathrm{~s} / 501$ it & $5203 \mathrm{~s} / 242$ it \\
\hline
\end{tabular}

These facts add to the overall computing time. Therefore we cannot expect a very dramatic decrease in computational time once the number of subdomains gets large. Nevertheless, we see in Table 4 that the domain decomposition algorithms for splittings with $D=4,8,16,32$ are still much faster than without decomposition $(D=1)$. In this case, for a nonoverlapping splitting into 8 domains the best performance is guaranteed, while for decomposing into 16 or more domains the algorithm already requires more time to reach its stopping criterion.

Splitting the image domain into larger subdomains, as happens for an overlapping decomposition, one may expect an increase in computational time. This may not necessarily occur, as the solution in the overlap is computed twice per iteration, which decreases the number of iterations. We even see in Table 4 that for a fixed number of subdomains the larger the overlapping region is, the fewer iterations are performed. In our numerical experiments we observe that for an overlapping decomposition into 16 domains with overlaps of size $50 \times 2576$ pixels the domain decomposition algorithm performs best with respect to the number of iterations and computational time.

We also observe that, with increasing number of subdomains, the number of iterations is decreasing. For a nonoverlapping decomposition the number of iterations is only decreasing very slowly, while for overlapping decompositions the decay is more noticeable. For the overlapping splitting, when doubling the number of domains, we see from Table 4 that for a larger overlap the absolute reduction of the number of iterations is larger than for a smaller overlap, while the relative reduction is bigger for smaller overlap.

6. Conclusion. We have proposed a combined $L^{1}-L^{2}$-TV functional with total variation regularization which outperforms the pure $L^{1}$-TV or $L^{2}$-TV models, as it preserves details better than the $L^{2}$-TV model and does not suffer from a sudden loss of image features like the $L^{1}$-TV model. Moreover, it is superior (in PSNR) in restoration tasks where images are corrupted simultaneously by Gaussian and salt-and-pepper noise.

For the numerical solution of the $L^{1}-L^{2}-\mathrm{TV}$ energy we have proposed and analyzed sequential and parallel subspace correction methods, which generate a convergent (sub)sequence of iterates and a monotone decrease of the energy. Moreover, we have shown that the distance between limit points and the global minimizer of the $L^{1}-L^{2}-\mathrm{TV}$ energy is bounded by the minimal norm of Lagrange multipliers associated with involved subspace projection constraints. In our numerical experiments this norm often appeared very small, or it might even 
tend to 0 , indicating that convergence to the global optimum was obtained. However, in the rare cases where the minimal norm of Lagrange multipliers did not tend to 0, we observed a resolution dependent effect reducing the multiplier norm under increasing resolution. This behavior may certainly motivate further research on the convergence of subspace correction methods for minimizing nonsmooth and nonadditive objectives. We have also shown that the parallel version pays off up to the number of subdomains where the communication between processors becomes dominant.

We also mention that the theoretical analysis of subspace correction methods for nonsmooth and nonadditive functionals is still far from being complete. In particular, in general Banach spaces there is not much known about such methods and their convergence to a global minimizer. Not even in a discrete setting for dimensions $d>1$ has this question yet been answered without invoking (rather restrictive) assumptions.

Appendix A. Proof of Proposition 4.5. The proof of Proposition 4.5 as stated here is similar to that in [50, Prop. 4.1]. It is clear that $\zeta \in \partial J_{\alpha_{1}, \alpha_{2}}(u)$ if and only if $u \in$ $\operatorname{argmin}_{v \in \mathcal{H}}\left\{J_{\alpha_{1}, \alpha_{2}}(v)-\langle\zeta, v\rangle_{\mathcal{H}}\right\}$; let us consider the following variational problem:

$(\mathcal{P})$

$\inf _{v \in \mathcal{H}}\left\{J_{\alpha_{1}, \alpha_{2}}(v)-\langle\zeta, v\rangle_{\mathcal{H}}\right\}=\inf _{v \in \mathcal{H}}\left\{\alpha_{1}\left\|T_{1} v-g_{1}\right\|_{\ell^{1}(\Omega)}+\alpha_{2}\left\|T_{2} v-g_{2}\right\|_{\ell^{2}(\Omega)}^{2}+\varphi(|\nabla v|)(\Omega)-\langle\zeta, v\rangle_{\mathcal{H}}\right\}$.

We denote such an infimum by $\inf (\mathcal{P})$. Now we compute $\left(\mathcal{P}^{*}\right)$, the dual of $(\mathcal{P})$. Let $\mathcal{F}: \mathcal{H} \rightarrow \mathbb{R}$, $\mathcal{G}: \mathcal{H}^{d} \times \mathcal{H} \times \mathcal{H} \rightarrow \mathbb{R}, \mathcal{G}_{0}: \mathcal{H}^{d} \rightarrow \mathbb{R}, \mathcal{G}_{1}: \mathcal{H} \rightarrow \mathbb{R}, \mathcal{G}_{2}: \mathcal{H} \rightarrow \mathbb{R}$ such that $\mathcal{F}(v)=$ $-\langle\zeta, v\rangle_{\mathcal{H}}, \mathcal{G}_{0}\left(w_{0}\right)=\varphi\left(\left|w_{0}\right|\right)(\Omega), \mathcal{G}_{1}(\bar{w})=\alpha_{1}\left\|w_{1}-g_{1}\right\|_{\ell^{1}(\Omega)}, \mathcal{G}_{2}(\bar{w})=\alpha_{2}\left\|w_{2}-g_{2}\right\|_{\ell^{2}(\Omega)}^{2}, \mathcal{G}(w)=$ $\mathcal{G}_{0}\left(w_{0}\right)+\mathcal{G}_{1}\left(w_{1}\right)+\mathcal{G}_{2}\left(w_{2}\right)$, with $w=\left(w_{0}, w_{1}, w_{2}\right) \in \mathcal{H}^{d} \times \mathcal{H} \times \mathcal{H}$. Then the dual problem of $(\mathcal{P})$ is given by (cf. $[26$, p. 60])

$$
\sup _{p^{*} \in \mathcal{H}^{d} \times \mathcal{H} \times \mathcal{H}}\left\{-\mathcal{F}^{*}\left(\Lambda^{*} p^{*}\right)-\mathcal{G}^{*}\left(-p^{*}\right)\right\},
$$

where $\Lambda: \mathcal{H} \rightarrow \mathcal{H}^{d} \times \mathcal{H} \times \mathcal{H}$ is defined by $\Lambda v=\left((\nabla v)^{1}, \ldots,(\nabla v)^{d}, T_{1} v, T_{2} v\right)$ and $\Lambda^{*}$ is its adjoint. We denote the supremum in $\left(\mathcal{P}^{*}\right)$ by $\sup \left(\mathcal{P}^{*}\right)$. Using the definition of the conjugate function, we compute $\mathcal{F}^{*}$ and $\mathcal{G}^{*}$. In particular, we have

$$
\mathcal{F}^{*}\left(\Lambda^{*} p^{*}\right)=\sup _{v \in \mathcal{H}}\left\{\left\langle\Lambda^{*} p^{*}, v\right\rangle_{\mathcal{H}}-\mathcal{F}(v)\right\}=\sup _{v \in \mathcal{H}}\left\langle\Lambda^{*} p^{*}+\zeta, v\right\rangle_{\mathcal{H}}= \begin{cases}0 & \text { if } \Lambda^{*} p^{*}+\zeta=0, \\ \infty & \text { otherwise, }\end{cases}
$$

where $p^{*}=\left(p_{0}^{*}, p_{1}^{*}, p_{2}^{*}\right)$, and due to the separability of $\mathcal{G}$ we find

$$
\begin{aligned}
\mathcal{G}^{*}\left(p^{*}\right) & =\sup _{w \in \mathcal{H}^{d} \times \mathcal{H} \times \mathcal{H}}\left\{\left\langle p^{*}, w\right\rangle_{\mathcal{H}^{d} \times \mathcal{H} \times \mathcal{H}}-\mathcal{G}(w)\right\} \\
& =\sup _{w_{0} \in \mathcal{H}}\left\{\left\langle p_{0}^{*}, w_{0}\right\rangle_{\mathcal{H}^{d}}-\mathcal{G}_{0}\left(w_{0}\right)\right\}+\sup _{w_{1} \in \mathcal{H}}\left\{\left\langle p_{1}^{*}, w_{1}\right\rangle_{\mathcal{H}}-\mathcal{G}_{1}\left(w_{1}\right)\right\}+\sup _{w_{2} \in \mathcal{H}}\left\{\left\langle p_{2}^{*}, w_{2}\right\rangle_{\mathcal{H}}-\mathcal{G}_{2}\left(w_{2}\right)\right\} \\
& =\mathcal{G}_{0}^{*}\left(p_{0}^{*}\right)+\mathcal{G}_{1}^{*}\left(p_{1}^{*}\right)+\mathcal{G}_{2}^{*}\left(p_{2}^{*}\right) .
\end{aligned}
$$

We have that $\mathcal{G}_{2}^{*}\left(p_{2}^{*}\right)=\left\langle\frac{p_{2}^{*}}{4 \alpha_{2}}+g_{2}, p_{2}^{*}\right\rangle_{\mathcal{H}}, \mathcal{G}_{1}^{*}\left(p_{1}^{*}\right)=\left\langle p_{1}^{*}, g_{1}\right\rangle_{\mathcal{H}}$ if $\left|p_{1}^{*}\right| \leq \alpha_{1}$, and (see [26]) $\mathcal{G}_{0}^{*}\left(p_{0}{ }^{*}\right)=\varphi_{+}^{*}\left(\left|p_{0}{ }^{*}\right|\right)(\Omega)$ if $\left|p_{0}^{*}(x)\right| \in \operatorname{Dom} \varphi_{+}^{*}$, where $\varphi_{+}^{*}$ is the conjugate function of $\varphi_{+}$ 
defined by $\varphi_{+}(t):=\varphi(|t|)$ for $t \in \mathbb{R}$. Therefore we can write $\left(\mathcal{P}^{*}\right)$ in the following way:

$$
\sup _{p^{*} \in \mathcal{K}}\left\{-\left\langle\frac{-p_{2}^{*}}{4 \alpha_{2}}+g_{2},-p_{2}^{*}\right\rangle_{\mathcal{H}}-\left\langle g_{1},-p_{1}^{*}\right\rangle_{\mathcal{H}}-\varphi_{+}^{*}\left(\left|p_{0}^{*}\right|\right)(\Omega)\right\},
$$

where $\mathcal{K}=\left\{p^{*} \in \mathcal{H}^{d} \times \mathcal{H} \times \mathcal{H}:\left|p_{0}{ }^{*}(x)\right| \in \operatorname{Dom} \varphi_{+}^{*}\right.$ and $\left.\left|p_{1}^{*}(x)\right| \leq \alpha_{1} \forall x \in \Omega, \Lambda^{*} p^{*}+\zeta=0\right\}$. The function $\varphi_{+}$also fulfills assumption $\left(A_{\varphi}\right)$ (ii) (i.e., there exist $c_{1}>0, b \geq 0$ such that $c_{+} z-b \leq \varphi_{+}(z) \leq c_{+} z+b$ for all $\left.z \in \mathbb{R}^{+}\right)$. The conjugate function of $\varphi_{+}$is given by $\varphi_{+}^{*}(t)=\sup _{z \in \mathbb{R}}\left\{\langle t, z\rangle-\varphi_{+}(z)\right\}$. Using the previous inequalities and the fact that $\varphi_{+}$is an even function (i.e., $\varphi_{+}(z)=\varphi_{+}(-z)$ for all $z \in \mathbb{R}$ ), we have

$$
\sup _{z \in \mathbb{R}}\left\{\langle t, z\rangle-c_{+}|z|+b\right\} \geq \sup _{z \in \mathbb{R}}\left\{\langle t, z\rangle-\varphi_{+}(z)\right\} \geq \sup _{z \in \mathbb{R}}\left\{\langle t, z\rangle-c_{1}|z|-b\right\}= \begin{cases}-b & \text { if }|t| \leq c_{1}, \\ \infty & \text { else. }\end{cases}
$$

In particular, one can see that $t \in \operatorname{Dom} \varphi_{+}^{*}$ if and only if $|t| \leq c_{1}$.

From $\Lambda^{*} p^{*}+\zeta=0$ we obtain

$$
\begin{aligned}
\left\langle\Lambda^{*} p^{*}, \omega\right\rangle_{\mathcal{H}}+\langle\zeta, \omega\rangle_{\mathcal{H}} & =\left\langle p^{*}, \Lambda \omega\right\rangle_{\mathcal{H}^{d+2}}+\langle\zeta, \omega\rangle_{\mathcal{H}} \\
& =\left\langle p_{0}^{*}, \nabla \omega\right\rangle_{\mathcal{H}^{d}}+\left\langle p_{1}^{*}, T_{1} \omega\right\rangle_{\mathcal{H}}+\left\langle p_{2}^{*}, T_{2} \omega\right\rangle_{\mathcal{H}}+\langle\zeta, \omega\rangle_{\mathcal{H}}=0 \quad \forall \omega \in \mathcal{H} .
\end{aligned}
$$

Then, since $\left\langle p_{0}{ }^{*}, \nabla \omega\right\rangle_{\mathcal{H}^{d}}=\left\langle-\operatorname{div} p_{0}^{*}, \omega\right\rangle_{\mathcal{H}}$ (see section 4.1), we have $T_{1}^{*} p_{1}^{*}+T_{2}^{*} p_{2}^{*}-\operatorname{div} p_{0}^{*}+\zeta=0$. Hence we can write $\mathcal{K}$ in the following way:

$$
\begin{gathered}
\mathcal{K}=\left\{p^{*}=\left(p_{0}^{*}, p_{1}^{*}, p_{2}^{*}\right) \in \mathcal{H}^{d} \times \mathcal{H} \times \mathcal{H}:\left|p_{0}{ }^{*}(x)\right| \leq c_{1} \text { and }\left|p_{1}^{*}(x)\right| \leq \alpha_{1} \forall x \in \Omega,\right. \\
\left.T_{1}^{*} p_{1}^{*}+T_{2}^{*} p_{2}^{*}-\operatorname{div} p_{0}^{*}+\zeta=0\right\} .
\end{gathered}
$$

We now apply the duality results from [26, Thm. III.4.1], since the objective functional in $(\mathcal{P})$ is convex, continuous with respect to $\Lambda v$ in $\mathcal{H}^{d} \times \mathcal{H} \times \mathcal{H}$, and $\inf (\mathcal{P})$ is finite. Consequently, $\inf (\mathcal{P})=\sup \left(\mathcal{P}^{*}\right) \in \mathbb{R}$, and $\left(\mathcal{P}^{*}\right)$ has a solution $M=\left(M_{0}, M_{1}, M_{2}\right) \in \mathcal{K}$.

Let us assume that $u$ is a solution of $(\mathcal{P})$ and $M$ is a solution of $\left(\mathcal{P}^{*}\right)$. From $\inf (\mathcal{P})=$ $\sup \left(\mathcal{P}^{*}\right)$ we get

$\alpha_{1}\left\|T_{1} u-g_{1}\right\|_{\ell^{1}(\Omega)}+\alpha_{2}\left\|T_{2} u-g_{2}\right\|_{\ell^{2}(\Omega)}^{2}+\varphi(|\nabla u|)(\Omega)-\langle\zeta, u\rangle_{\mathcal{H}}$

$$
=-\left\langle\frac{-M_{2}}{4 \alpha_{2}}+g_{2},-M_{2}\right\rangle_{\mathcal{H}}-\left\langle g_{1},-M_{1}\right\rangle_{\mathcal{H}}-\varphi_{1}^{*}\left(\left|M_{0}\right|\right)(\Omega),
$$

where $M=\left(M_{0}, M_{1}, M_{2}\right) \in \mathcal{H}^{d} \times \mathcal{H} \times \mathcal{H},\left|M_{0}(x)\right| \leq c_{1},\left|M_{1}\right| \leq \alpha_{1}$, and $T_{1}^{*} M_{1}+T_{2}^{*} M_{2}-$ $\operatorname{div} M_{0}+\zeta=0$, which verifies (4.8). In particular, (A.3) and (A.4) yield

$$
\begin{gathered}
\alpha_{1}\left\|T_{1} u-g_{1}\right\|_{\ell^{1}(\Omega)}+\alpha_{2}\left\|T_{2} u-g_{2}\right\|_{\ell^{2}(\Omega)}^{2}+\varphi(|\nabla u|)(\Omega)+\left\langle M_{1}, T_{1} u\right\rangle_{\mathcal{H}}+\left\langle M_{2}, T_{2} u\right\rangle_{\mathcal{H}} \\
+\left\langle M_{0}, \nabla u\right\rangle_{\mathcal{H}^{d}}+\left\langle\frac{-M_{2}}{4 \alpha_{2}}+g_{2},-M_{2}\right\rangle_{\mathcal{H}}+\left\langle g_{1},-M_{1}\right\rangle_{\mathcal{H}}+\varphi_{1}^{*}\left(\left|M_{0}\right|\right)(\Omega)=0 .
\end{gathered}
$$


We rewrite (A.5) in the following form:

(A.6)

$$
\begin{aligned}
& \sum_{x \in \Omega} \alpha_{1}\left|\left(T_{1} u-g_{1}\right)(x)\right|+\sum_{x \in \Omega} \alpha_{2}\left|\left(T_{2} u-g_{2}\right)(x)\right|^{2}+\sum_{x \in \Omega} \varphi(|(\nabla u)(x)|)+\sum_{x \in \Omega} M_{1}(x)\left(T_{1} u\right)(x) \\
& \quad+\sum_{x \in \Omega} M_{2}(x)\left(T_{2} u\right)(x)+\sum_{x \in \Omega}\left\langle M_{0}(x), \nabla u(x)\right\rangle_{\mathbb{R}^{d}}-\sum_{x \in \Omega}\left(\frac{-M_{2}(x)}{4 \alpha_{2}}+g_{2}(x)\right)\left(M_{2}\right)(x) \\
& \quad+\sum_{x \in \Omega} g_{1}(x)\left(-M_{1}\right)(x)+\sum_{x \in \Omega} \varphi_{1}^{*}\left(\left|M_{0}(x)\right|\right)=0 .
\end{aligned}
$$

Now for the various terms in (A.6) we have the following:

1. $\alpha_{1}\left|\left(T_{1} u-g_{1}\right)(x)\right|+M_{1}(x)\left(\left(T_{1} u\right)(x)-g_{1}(x)\right) \geq 0$ since $\left|M_{1}(x)\right| \leq \alpha_{1}$.

2. $\alpha_{2}\left|\left(T_{2} u-g_{2}\right)(x)\right|^{2}+M_{2}(x)\left(T_{2} u(x)-g_{2}(x)\right)+\frac{M_{2}(x)^{2}}{4 \alpha_{2}}=\left(\sqrt{\alpha_{2}}\left(T_{2} u-g_{2}\right)(x)+\frac{M_{2}(x)}{2 \sqrt{\alpha_{2}}}\right)^{2} \geq 0$.

3. $\varphi(|(\nabla u)(x)|)+\left\langle M_{0}(x), \nabla u(x)\right\rangle_{\mathbb{R}^{d}}+\varphi_{1}^{*}\left(\left|M_{0}(x)\right|\right) \geq \varphi(|(\nabla u)(x)|)-\sum_{j=1}^{d}\left|M_{0}^{j}(x)\right|\left|S^{j}\right|+$ $\varphi_{1}^{*}\left(\left|M_{0}(x)\right|\right) \geq 0$ by the definition of $\varphi_{1}^{*}$, since

$$
\varphi_{1}^{*}\left(\left|M_{0}(x)\right|\right)=\sup _{S=\left(S^{1}, \ldots, S^{d}\right) \in \mathbb{R}^{d}}\left\{\sum_{j=1}^{d}\left|M_{0}^{j}(x)\right|\left|S^{j}\right|-\varphi(|S|)\right\} .
$$

Hence, condition (A.6) reduces to

$$
\begin{aligned}
\varphi(|(\nabla u)(x)|)+\left\langle M_{0}(x), \nabla u(x)\right\rangle_{\mathbb{R}^{d}}+\varphi_{1}^{*}\left(\left|M_{0}(x)\right|\right)=0 & \forall x \in \Omega, \\
M_{2}(x)=-2 \alpha_{2}\left(T_{2} u-g_{2}\right)(x) & \forall x \in \Omega, \\
\alpha_{1}\left|\left(T_{1} u-g_{1}\right)(x)\right|+M_{1}(x)\left(\left(T_{1} u\right)(x)-g_{1}(x)\right)=0 & \forall x \in \Omega .
\end{aligned}
$$

Conversely, if there exists $M=\left(M_{0}, M_{1}, M_{2}\right) \in \mathcal{H}^{d} \times \mathcal{H} \times \mathcal{H}$ with $\left|M_{0}(x)\right| \leq c_{1}$ and $\left|M_{1}\right| \leq$ $\alpha_{1}$, which fulfills conditions (4.5) and (4.8), then it is clear from our previous considerations that (A.4) holds. Let us denote the functional on the left-hand side of (A.4) by

$$
P(u):=\alpha_{1}\left\|T_{1} u-g_{1}\right\|_{\ell^{1}(\Omega)}+\alpha_{2}\left\|T_{2} u-g_{2}\right\|_{\ell^{2}(\Omega)}^{2}+\varphi(|\nabla u|)(\Omega)-\langle\zeta, u\rangle_{\mathcal{H}}
$$

and the functional on the right-hand side of (A.4) by

$$
P^{*}(M):=-\left\langle\frac{-M_{2}}{4 \alpha_{2}}+g_{2},-M_{2}\right\rangle_{\mathcal{H}}-\left\langle g_{1},-M_{1}\right\rangle_{\mathcal{H}}-\varphi_{1}^{*}\left(\left|M_{0}\right|\right)(\Omega) .
$$

Hence $\inf P=\inf (\mathcal{P})$ and $\sup P^{*}=\sup \left(\mathcal{P}^{*}\right)$. Since $P$ is convex, continuous with respect to $\Lambda u$ in $\mathcal{H}^{d} \times \mathcal{H} \times \mathcal{H}$, and $\inf (\mathcal{P})$ is finite, we know from duality results [26, Thm. III.4.1] that $\inf (\mathcal{P})=\sup \left(\mathcal{P}^{*}\right) \in \mathbb{R}$. We assume that $M$ is no solution of $\left(\mathcal{P}^{*}\right)$, i.e., $P^{*}(M)<\sup \left(\mathcal{P}^{*}\right)$, and $u$ is no solution of $(\mathcal{P})$, i.e., $P(u)>\inf (\mathcal{P})$. Then we have that $P(u)>\inf (\mathcal{P})=\sup \left(\mathcal{P}^{*}\right)>$ $P^{*}(M)$. Thus (A.4) is valid if and only if $M$ is a solution of $\left(\mathcal{P}^{*}\right)$ and $u$ is a solution of $(\mathcal{P})$, which is equivalent to $\zeta \in \partial J_{\alpha_{1}, \alpha_{2}}(u)$.

If, additionally, $\varphi$ is differentiable and $|(\nabla u)(x)| \neq 0$ for $x \in \Omega$, then $M_{0}(x)$ can be computed explicitly. In fact, from (A.7) (respectively, (4.5)) we have

$$
\varphi_{1}^{*}\left(\left|-M_{0}(x)\right|\right)=-\left\langle M_{0}(x),(\nabla u)(x)\right\rangle_{\mathbb{R}^{d}}-\varphi(|(\nabla u)(x)|) .
$$


From the definition of conjugate functions we have

$$
\begin{aligned}
\varphi_{1}^{*}\left(\left|-M_{0}(x)\right|\right) & =\sup _{t \in \mathbb{R}}\left\{\left|-M_{0}(x)\right| t-\varphi_{1}(t)\right\}=\sup _{t \geq 0}\left\{\left|-M_{0}(x)\right| t-\varphi_{1}(t)\right\} \\
& =\sup _{t \geq 0} \sup _{\substack{S \in \mathbb{R}^{d} \\
|S|=t}}\left\{\left\langle-M_{0}(x), S\right\rangle_{\mathbb{R}^{d}}-\varphi_{1}(|S|)\right\}=\sup _{S \in \mathbb{R}^{d}}\left\{\left\langle-M_{0}(x), S\right\rangle_{\mathbb{R}^{d}}-\varphi(|S|)\right\} .
\end{aligned}
$$

Now, if $|(\nabla u)(x)| \neq 0$ for $x \in \Omega$, then it follows from (A.10) that the supremum is taken in $S=|(\nabla u)(x)|$ and we have $\nabla_{S}\left(-\left\langle M_{0}(x), S\right\rangle_{\mathbb{R}^{d}}-\varphi(|S|)\right)=0$, which implies

$$
M_{0}^{j}(x)=-\frac{\varphi^{\prime}(|(\nabla u)(x)|)}{|(\nabla u)(x)|}(\nabla u)^{j}(x), \quad j=1, \ldots, d,
$$

and verifies (4.9). This finishes the proof.

\section{REFERENCES}

[1] S. Aliney, A property of the minimum vectors of a regularizing functional defined by means of absolute norm, IEEE Trans. Signal Process., 45 (1997), pp. 913-917.

[2] L. Ambrosio, N. Fusco, And D. Pallara, Functions of Bounded Variation and Free Discontinuity Problems, Oxford Math. Monogr., Clarendon Press, Oxford, 2000.

[3] H. Attouch, G. Buttazzo, and G. Michaille, Variational Analysis in Sobolev and BV Spaces: Applications to PDEs and Optimization, MPS-SIAM Ser. Optim. 6, SIAM, Philadelphia, 2006.

[4] J.-F. Aujol, G. Gilboa, T. Chan, And S. Osher, Structure-texture image decomposition-Modeling, algorithms, and parameter selection, Int. J. Comput. Vision, 67 (2006), pp. 111-136.

[5] D. Bertsekas, Constrained Optimization and Lagrange Multiplier Methods, Academic Press, New York,1982.

[6] D. Bertsekas, A. Nedić, and A. Ozdaglar, Convex Analysis and Optimization, Athena Scientific, Belmont, MA, 2003.

[7] A. Braides, Г-Convergence for Beginners, Oxford Lecture Ser. Math. Appl. 22, Oxford University Press, New York, 2002.

[8] L. BREgman, A relaxation method of finding a common point of convex sets and its application to the solution of convex programming problems. J. Wych. Math. Math. Phys., 7 (1967), pp. 200-217.

[9] J. F. Cai, R. Chan, and M. Nikolova, Two phase methods for deblurring images corrupted by impulse plus Gaussian noise, AIMS J. Inverse Prob. Imaging, 2 (2008), pp. 187-204.

[10] C. Carstensen, Domain decomposition for a non-smooth convex minimization problem and its application to plasticity, Numer. Linear Algebra Appl., 4 (1998), pp. 177-190.

[11] A. Chambolle, An algorithm for total variation minimization and applications. J. Math. Imaging Vision, 20 (2004), pp. 89-97.

[12] A. Chambolle and J. Darbon, On total variation minimization and surface evolution using parametric maximum flows, Int. J. Comput. Vis., 84 (2009), pp. 288-307.

[13] A. Chambolle and P.-L. Lions, Image recovery via total variation minimization and related problems, Numer. Math., 76 (1997), pp. 167-188.

[14] R. H. Chan, Y. Dong, And M. Hintermüller, An efficient two-phase L1-TV method for restoring blurred images with impulse noise, IEEE Trans. Image Process., 19 (2010), pp. 1731-1739.

[15] R. H. Chan, C.-W. Ho, AND M. Nikolova, Salt-and-pepper noise removal by median-type noise detectors and details-preserving regularization, IEEE Trans. Image Process., 14 (2005), pp. 1479-1485.

[16] T. F. Chan And S. Esedō̄Lu, Aspects of total variation regularized $L^{1}$ function approximation, SIAM J. Appl. Math., 65 (2005), pp. 1817-1837.

[17] T. F. Chan, G. H. Golub, and P. Mulet, A nonlinear primal-dual method for total variation-based image restoration, SIAM J. Sci. Comput., 20 (1999), pp. 1964-1977.

[18] T. F. Chan and T. P. Mathew, Domain decomposition algorithms, Acta Numer., 3 (1994), pp. 61-143. 
[19] P. L. Combettes And V. R. WAJs, Signal recovery by proximal forward-backward splitting, Multiscale Model. Simul., 4 (2005), pp. 1168-1200.

[20] J. Darbon And M. Sigelle, A fast and exact algorithm for total variation minimization, in Proceedings of the IbPRIA 2005, Lecture Notes in Comput. Sci. 3522, Springer, New York, 2005, pp. 351-359.

[21] J. Darbon And M. Sigelle, Image restoration with discrete constrained total variation Part I: Fast and exact optimization, J. Math. Imaging Vision, 26 (2006), pp. 261-276.

[22] I. Daubechies, G. Teschke, and L. Vese, Iteratively solving linear inverse problems under general convex constraints, Inverse Problems Imaging, 1 (2007), pp. 29-46.

[23] J. Delon And A. Desolneux, A patch-based approach for removing impulse or mixed Gaussian-impulse noise, SIAM J. Imaging Sci., 6 (2013), pp. 1140-1174.

[24] D. C. Dobson And C. R. Vogel, Convergence of an iterative method for total variation denoising, SIAM J. Numer. Anal., 34 (1997), pp. 1779-1791.

[25] Y. Dong, M. Hintermüller, And M. Neri, An efficient primal-dual method for $L^{1}$ TV image restoration, SIAM J. Imaging Sci., 2 (2009), pp. 1168-1189.

[26] I. Ekeland and R. Temam, Convex Analysis and Variational Problems, Stud. Math. Appl. 1, NorthHolland, Amsterdam, Elsevier, New York, 1976.

[27] M. FornAsier, Domain decomposition methods for linear inverse problems with sparsity constraints, Inverse Problems, 23 (2007), pp. 2505-2526.

[28] M. Fornasier, Y. Kim, A. Langer, And C.-B. Schönlieb, Wavelet decomposition method for $L_{2} / T V-$ image deblurring, SIAM J. Imaging Sci., 5 (2012), pp. 857-885.

[29] M. Fornasier, A. LAnger, And C.-B. SchÖnlieb, A convergent overlapping domain decomposition method for total variation minimization, Numer. Math., 116 (2010), pp. 645-685.

[30] M. FornAsier AND C.-B. SchönliEB, Subspace correction methods for total variation and $\ell_{1}$ minimization, SIAM J. Numer. Anal., 47 (2009), pp. 3397-3428.

[31] R. Garnett, T. Huegerich, C. Chui, And W. He, A universal noise removal algorithm with an impulse detector, IEEE Trans. Image Process., 14 (2005), pp. 1747-1754.

[32] T. Goldstein And S. Osher, The split Bregman method for L1-regularized problems, SIAM J. Imaging Sci., 2 (2009), pp. 323-343.

[33] M. Hintermüller AND K. Kunisch, Total bounded variation regularization as bilaterally constrained optimization problem, SIAM J. Appl. Math., 64 (2004), pp. 1311-1333.

[34] M. Hintermüller and G. Stadler, An infeasible primal-dual algorithm for total bounded variationbased inf-convolution-type image restoration, SIAM J. Sci. Comput., 28 (2006), pp. 1-23.

[35] J.-B. Hiriart-Urruty and C. Lemaréchal, Convex Analysis and Minimization Algorithms I, Grundlehren Math. Wiss. 305, Springer-Verlag, Berlin, 1996.

[36] K. Ito And K. Kunisch, Lagrange Multiplier Approach to Variational Problems and Applications, Adv. Des. Control 15, SIAM, Philadelphia, 2008.

[37] A. Langer, Subspace Correction and Domain Decomposition Methods for Total Variation Minimization, Ph.D. thesis, Johannes Kepler University Linz, Linz, Austria, 2011.

[38] A. Langer, S. Osher, And C.-B. Schönlieb, Bregmanized domain decomposition for image restoration, J. Sci. Comput., 54 (2013), pp. 549-576.

[39] Y. MeYer, Oscillating Pattern in Image Processing and Nonlinear Evolution Equations, AMS University Lecture Series 22, AMS, Providence, RI, 2001.

[40] Y. Nesterov, Smooth minimization of non-smooth functions, Math. Program. Ser. A, 103 (2005), pp. $127-152$.

[41] M. Nikolova, Minimizers of cost-functions involving nonsmooth data-fidelity terms. Application to the processing of outliers, SIAM J. Numer. Anal., 40 (2002), pp. 965-994.

[42] M. Nikolova, A variational approach to remove outliers and impulse noise, J. Math. Imaging Vision, 20 (2004), pp. 99-120.

[43] S. Osher, M. Burger, D. Goldfarb, J. Xu, And W. Yin, An iterative regularization method for total variation-based image restoration, Multiscale Model. Simul., 4 (2005), pp. 460-489.

[44] R. T. Rockafellar, Convex Analysis, Princeton University Press, Princeton, NJ, 1970.

[45] L. I. Rudin, S. Osher, And E. FAtemi, Nonlinear total variation based noise removal algorithms, Phys. D, 60 (1992), pp. 259-268.

[46] X.-C. TAi And P. Tseng, Convergence rate analysis of an asynchronous space decomposition method for convex minimization, Math. Comp., 71 (2001), pp. 1105-1135. 
[47] X.-C. TAI AND J. XU, Global convergence of subspace correction methods for convex optimization problems, Math. Comp., 71 (2002), pp. 105-124.

[48] P. Tseng, Convergence of a block coordinate descent method for nondifferentiable minimization, J. Optim. Theory Appl., 109 (2001), pp. 475-494.

[49] P. Tseng And S. Yun, A coordinate gradient descent method for nonsmooth separable minimization, Math. Program. J. Ser. B, 117 (2009), pp. 387-423.

[50] L. VESE, A study in the BV space of a denoising-deblurring variational problem., Appl. Math. Optim., 44 (2001), pp. 131-161.

[51] C. VONESCH AND M. UnSER, A fast multilevel algorithm for wavelet-regularized image restoration, IEEE Trans. Image Process., 18 (2009), pp. 509-523.

[52] J. Warga, Minimizing certain convex functions, J. Soc. Indust. Appl. Math., 11 (1963), pp. 588-593.

[53] P. Weiss, L. Blanc-FÉRAud, ANd G. Aubert, Efficient schemes for total variation minimization under constraints in image processing, SIAM J. Sci. Comput., 31 (2009), pp. 2047-2080.

[54] J. Xu, The method of subspace corrections, J. Comput. Appl. Math., 128 (2001), pp. 335-362.

[55] W. Yin, S. Osher, D. Goldfarb, And J. Darbon, Bregman iterative algorithms for $\ell_{1}$-minimization with applications to compressed sensing, SIAM J. Imaging Sci., 1 (2008), pp. 143-168.

[56] X. Zhang, M. Burger, X. Bresson, And S. Osher, Bregmanized nonlocal regularization for deconvolution and sparse reconstruction, SIAM J. Imaging Sci., 3 (2010), pp. 253-276.

[57] M. Zhu and T. Chan, An Efficient Primal-Dual Hybrid Gradient Algorithm for Total Variation Image Restoration, CAM Reports 08-34, Center for Applied Math., UCLA, Los Angeles, CA, 2008. 